ournal for ImmunoTherapy of Cancer

\section{A new oncolytic Vaccinia virus augments antitumor immune responses to prevent tumor recurrence and metastasis after surgery}

To cite: Ahmed J, Chard LS, Yuan M, et al. A new oncolytic Vaccinia virus augments antitumor immune responses to prevent tumor recurrence and metastasis after surgery. Journal for ImmunoTherapy of Cancer 2020;8:e000415. doi:10.1136/jitc-2019-000415

- Additional material is published online only. To view please visit the journal online (http://dx.doi.org/10.1136/jitc2019-000415).

JA, LSC and MY contributed equally.

Accepted 19 December 2019

Check for updates

(c) Author(s) (or their employer(s)) 2020. Re-use permitted under CC BY. Published by BMJ.

${ }^{1}$ Centre for Biomarkers \& Biotherapeutics, Barts Cancer Institute, Queen Mary University of London, London, UK

${ }^{2}$ National Centre for International Research in Cell and Gene Therapy, Zhengzhou University, Zhengzhou, Henan, China

${ }^{3}$ University of Illinois at Chicago, Chicago, Illinois, USA

Correspondence to

Dr Yaohe Wang;

yaohe.wang@qmul.ac.uk

\section{ABSTRACT}

Background Local recurrence and remote metastasis are major challenges to overcome in order to improve the survival of patients with cancer after surgery. Oncolytic viruses are a particularly attractive option for prevention of postsurgical disease as they offer a non-toxic treatment option that can directly target residual tumor deposits and beneficially modulate the systemic immune environment that is suppressed post surgery and allows residual disease escape from control. Here, we report that a novel Vaccinia virus (VV), $\mathrm{V} \Delta \mathrm{TK} \Delta \mathrm{N} 1 \mathrm{~L}$ (with deletion of both thymidine kinase (TK) and N1L genes) armed with interleukin 12 (IL-12), can prolong postoperative survival when used as a neoadjuvant treatment in different murine and hamster surgical models of cancer.

Methods A tumor-targeted replicating W with deletion of TK gene and N1L gene (VV $\Delta \mathrm{TK} \Delta \mathrm{N} 1 \mathrm{~L})$ was created. This virus was armed rationally with IL-12. The effect of $\mathrm{V} \Delta \Delta T K \Delta N 1 \mathrm{~L}$ and $\mathrm{V} \Delta \Delta \mathrm{TK} \Delta \mathrm{N} 1 \mathrm{~L}-\mathrm{IL} 12$ on modulation of the tumor microenvironment and induction of tumorspecific immunity as well the feasibility and safety as a neoadjuvant agent for preventing recurrence and metastasis after surgery were assessed in several clinically relevant models.

Results WVTK $\Delta \mathrm{N} 1 \mathrm{~L}$ can significantly prolong postoperative survival when used as a neoadjuvant treatment in three different surgery-induced metastatic models of cancer. Efficacy was critically dependent on elevation of circulating natural killer cells that was achieved by virus-induced cytokine production from cells infected with N1L-deleted, but not N1L-intact V. This effect was further enhanced by arming $\mathrm{V} \Delta \Delta \mathrm{TK} \Delta \mathrm{N} 1 \mathrm{~L}$ with $\mathrm{IL}-12$, a potent antitumor cytokine. Five daily treatments with VV $\Delta T K \Delta$ N1L-IL12 before surgery dramatically improved postsurgical survival. WV $\Delta \mathrm{TK} \Delta \mathrm{N} 1 \mathrm{~L}$ armed with human IL-12 completely prevented tumor recurrence in surgical models of head and neck cancer in Syrian hamsters.

Conclusions These data provide a proof of concept for translation of the regime into clinical trials. W $\Delta T K \Delta N 1 \mathrm{~L}-$ IL12 is a promising agent for use as an adjuvant to surgical treatment of solid tumors.

\section{INTRODUCTION}

The success of immune checkpoint inhibition at achieving durable remission in a number of cancers has led to a recent evolution in cancer therapy to include immunotherapeutic options as mainstay treatments. Oncolytic viruses (OV) are emerging as a powerful class of immunotherapeutic with the potential to act in synergy with other treatment modalities to improve clinical outcome. Indeed, the recent approval of oncolytic Herpes simplex virus, talimogene laherparepvec (T-VEC), has paved the way in ackowledging OV as validated therapeutics. ${ }^{1}$ OV take advantage of a number of changes in the tumor microenvironment (TME) to facilitate vascular collapse, immunogenic lysis of cancer cells and augmentation of antitumor immune responses, which are often absent in the TME. With the success of T-VEC, we are now moving toward creating the optimal viral vector to maximize the anticancer potency, which can act as stand-alone treatments or complement traditional therapies such as surgical removal of primary tumors.

Surgical excision remains the principle treatment option for solid tumors; however, clinical and experimental data suggest that tumor excision may in fact promote tumor recurrence and metastasis. ${ }^{2}$ The main source of this recurrence is minimal residual disease (MRD) left in situ in microscopic deposits beyond the clearance margins. ${ }^{3}$ A number of mechanisms have been proposed to explain the altered growth of MRD after surgery, including direct dissemination of the tumor cells into the circulatory and lymphatic systems during the procedure, a disinhibition of angiogenesis postoperatively ${ }^{4}$ and the perturbation of the immune system generated 
by surgical intervention, including marked Th2 polarization and natural killer (NK) cell dysfunction, generating a window of opportunity for tumor immune escape. ${ }^{25} \mathrm{NK}$ cells are innate immune cytotoxic lymphocytes that have long been implicated in the control of tumor growth and metastasis . An increasing number of clinical and experimental studies directly implicate postsurgical NK dysfunction in metastatic spread of MRD. ${ }^{6}$ Restoration of NK cell function within this window of opportunity therefore represents an important priority for prevention of postoperative recurrence.

Incorporating $\mathrm{OV}$ therapy into traditional surgical regimes is an attractive therapeutic option as replicationcompetent OVs such as Vaccinia virus (VV) may be able to directly target and eliminate remaining tumor deposits. OVs are powerful stimulants of antitumor immunity ${ }^{7}$ that can promote long-term tumor immune surveillance ${ }^{8}$ by activating both NK cells and tumor-specific cytotoxic T lymphocytes (CTL), critical antitumor immune effectors that are additionally dysregulated post surgery. ${ }^{9}$ Indeed, it was recently demonstrated that presurgical administration of pox virus could reverse NK cell suppression in experimental models of breast cancer and melanoma in addition to human patients, but the efficacy was limited. ${ }^{6}$ In addition, VV-based OV therapies have strong potential within the framework of postsurgical immune restoration because in addition to the attributes of OV discussed, we have shown that VV entry into tumor cells is facilitated by vascular endothelial growth factor, ${ }^{10}$ levels of which are elevated following surgical stress. ${ }^{11}$

Given the role of NK cells in containing disease, in particular targeting tumor cell populations that are refractory to adaptive immune control via MHCI suppression, and reducing postoperative morbidity, we sought to rationally redesign our thymidine kinase (TK)-deleted Lister strain VV, Vaccinia Virus Lister 15 (VVL15) ${ }^{12}$ to interrupt naturally evolved viral mechanisms of antiviral NK cell suppression with a view to creating a more powerful vector for primary treatment of cancers and additionally optimized as a neoadjuvant therapy. The VV N1L protein is a $13.8 \mathrm{KDa}$, non-essential virulence determinant ${ }^{13}$ and plays an important role in immune evasion via inhibition of cellular inflammatory pathways and early innate immune responses against viral infection, in particular NK cell activity. ${ }^{14}$ VVs engineered to lack N1L have previously been shown to be attenuated in mice. ${ }^{15}$ N1L inhibits NF- $\kappa B$ signalling in infected cells and deletion has been demonstrated to elevate NK cell responses to viral infection ${ }^{14}$ and improve generation of immediate and long-term memory CD8 +T cell responses, ${ }^{16}$ both of which could be expected to vastly improve the immunotherapeutic potential of oncolytic VV. Here, we demonstrate that intratumoral (i.t.) delivery of N1L-deleted VVL15 (VVL $\Delta$ TK $\Delta$ N1L) can control disease and extend survival in subcutaneous models of pancreatic cancer in a $\mathrm{T}$ cell-dependent manner. Additionally, by engaging innate immune responses, VVL $\Delta \mathrm{TK} \Delta \mathrm{N} 1 \mathrm{~L}$ can reduce metastatic spread from primary tumors and prolong postoperative survival in more aggressive murine models of cancer via upregulation of inflammatory cytokines and circulating NK cells. Efficacy was further enhanced by localized, OV-mediated delivery of the cytokine interleukin 12 (IL-12), consistently demonstrated as one of the most potent antitumor cytokines. ${ }^{17}$

\section{MATERIALS AND METHODS Study approval}

All mouse studies were carried out under the terms of the Home Office Project Licence PPL 70/6030 and subject to Queen Mary University of London ethical review, according to the guidelines for the welfare and use of animals in cancer research. Surgical experiments for efficacy studies of 4T1 and LY2 models as well as all hamster procedures were approved by the Animal Welfare and Research Ethics Committee of Zhengzhou University (Zhengzhou, China). For in vivo experiments, power calculations were carried out to determine required sample sizes using $G^{*}$ Power 3 , setting parameters of $\alpha=0.1$, power $=90 \%$, effect size $=30 \%$, and group $=3$. In subcutaneous tumor models, animals were assigned to treatment groups by matching tumor sizes prior to treatment. Tumor growth was measured using electronic callipers until tumors measured $1.4 \mathrm{~cm}$ (mice) or $1.8 \mathrm{~cm}$ (hamster) in diameter or ulcerated, at which point the animals were sacrificed. Tumor growth curves were terminated on the death of the first animal in each group, but group survival was monitored until the experimental endpoint.

\section{Cell lines}

CT26 (metastatic colon adenocarcinoma, BALB/c), CMT93 (rectal adenocarcinoma, C57BL/6), Lewis lung carcinoma (LLC, metastatic lung squamous cell carcinoma, C57BL/6) and B16-F10 (metastatic melanoma, C57BL/6) were obtained from CRUK, Clare Hall, Herts, UK. SCCVII is a murine oral cavity squamous carcinoma cell line $(\mathrm{C} 3 \mathrm{H} / \mathrm{HeN}$ strain $)$ and was a kind gift of Dr Osam Mazda (Department of Microbiology, Kyoto Prefectural University of Medicine, Kyoto City, Japan). DT6606, a pancreatic ductal adenocarcinoma (PDAC) cell line, derived from LSL-KrasG12D/+; Pdx-1-Cre mice which was a kind gift of Professor David Tuveson (CRUK, Cambridge Research Institute, Cambridge, UK). CV1 (African monkey kidney) cells were obtained from ATCC. LY-2 cell line was isolated from lymph node metastasis after inoculation with PAM212 squamous cell carcinoma cells. They were kindly provided by Dr Carter Van Waes (National Institute of Health, Bethesda, MD, USA). HCPC1 cell line was established from an epidermoid carcinoma of the Syrian golden hamster cheek and was generously provided by Professor Joel Schwartz of University of Illinois.

\section{Viruses}

$\mathrm{VV} \Delta \mathrm{TK} \Delta \mathrm{N} 1 \mathrm{~L}$ was created using the TK-deleted VVL15 platform. ${ }^{18}$ The N1L right arm was amplified from viral 
DNA using the primers RA_F: 5’ AAGCTTACGCGTATCT AATAAGTAGAGTCCTCATGCT where HindIII and MluI restriction sites are underlined and RA-R: 5' GGATCC CGGAAGGTAGTAGCATGGA where the BamHI restriction site is underlined. This fragment was cloned into the multiple cloning region of pUC19 (NEB) via HindIII and BamHI. A red fluorescent protein (RFP) vector with H5 promoter ${ }^{19} 20$ was constructed in pGEM-T (Promega) by amplification of RFP from pCMVdsRedExpress2 (Clonetech Labs) using the primers H5RFP_F: 5' CCGCGGAAAA ATTGAAAATAAATACAAAGGTTCTTGAGGGTTGTGT TAAATTGAAAGGGAGAAATAATCATAAATAGCTACCGGACTCAGATCCAC where the SacII restriction site is underlined and the $\mathrm{H} 5$ promoter shown in bold. H5RFP_R: 5' ACGCGTATTTAAATAAGCTTTATTTATG ATTATTTCTCGCTTTCAATTTAACACAACCCTCAAG AACCTTTGTATTTATTTTCAATTTTTCGCCTTAAGATACATTGATG where HindIII, SwaI and MluI restriction sites are underlined and a second $\mathrm{H} 5$ promoter is shown in bold to create a subclone H5-RFP-H5. The left arm of N1L was amplified from viral DNA and subcloned into PGEM-T using the primers LA_F: 5' AAGCTTGTCCTATCGTAGGCGATAGA where the HindII restriction site is underlined and the reverse primer LA-R: 5' GTCGACTATTTATG ATTATTTCTCGCTTTCAATTTAACACAACCCTCAA GAACCTTTGTATTTATTTTCAATTTTTGATCTAAT GATTGATCTATATGGTG where SalI is underlined and a third $\mathrm{H} 5$ promoter is shown in bold to create the subclone Left Arm-H5. H5-RFP-H5 was released using SacII and MluI and Left Arm-H5 was released using SalI and HindII. The pUC19 right arm construct was digested with HindII and MluI and the two inserts cloned into this vector to create an N1L shuttle vector; N1L Left Arm-H5H5-RFP-H5-Right Arm. Virus recombination and production were carried out as described previously. ${ }^{80}$ Murine and human IL-12 (for use in mouse and hamster models, respectively) were amplified from pUNO1-mIL12 and pUNO1-hIL12 (Invivogen) using the primers mIL12_F: 5' AAGCTTATGTGTCCTCAGAAGCTAACCATCTC where HindIII is underlined and mIL12_R: $5^{\prime}$ ATTTAAAT CCATACCACATTTGTAGAGG where SwaI is underlined or hIL12_F: 5' AAGCTTATGTGTCACCAGCAGTTGG and hIL12_R: 5' ATTTAAACCATACCACATTTGTAGAGG. These fragments were inserted into the HindII/ SwaI-digested H5-RFP-H5 subclone prior to creation of the entire shuttle vector to create N1L Left Arm-H5-H5RFP-H5-m/hIL-21-Right Arm.

\section{Viral replication and cell cytotoxicity assays}

Cell lines were infected with virus at a multiplicity of infection (MOI) of 1 plaque forming units (PFU)/cell. Cells and supernatant were collected at 24, 48 and 72 hours post infection. The median tissue culture infective dose (TCID50) for each sample was calculated as previously described. ${ }^{8}$ The cytotoxicity of the viruses in each cell line was assessed 6 days following infection using an MTS cell proliferation assay kit (Promega), according to the manufacturer's instructions.
ELISA

Cytokines/chemokines levels were detected by ELISAs in accordance with the manufacturer's instructions. Granulocyte-macrophage colony-stimulating factor (GMCSF), IL1 $\alpha$, IL1 $\beta$, mIL12, hIL12 (Biolegend); granulocyte colony-stimulating factor (GCSF), IL18, keratinocyte chemoattractant (KC; CXCL1) (R\&D Systems); macrophage inflammatory protein (MIP)-1 $\alpha$ (eBioscience). Murine inflammatory cytokines/chemokines in homogenized tumor tissue were obtained using Qiagen Multi-analyte ELISArray kits.

\section{Immunophenotyping cells}

Single-cell suspensions of tumor or spleen and virally infected antigen-presenting cells (APCs) and macrophages were prepared in fluorescence-activated cell sorting (FACS) buffer (FB; phosphate-buffered saline (PBS) containing $1 \%$ heat-inactivated bovine calf serum (BCS)). All fluorophore-conjugated antibodies used at 1:200 dilution and supplied by eBioscience. Cells were blocked with anti-CD16/32 prior to incubation with flurophore-conjugated antibody for $30 \mathrm{~min}$. Cells were fixed in $2 \%$ formalin and analyzed using an LSRFortessa multichannel flow cytometer (Beckton Dickinson (BD) Biosciences). Raw data were analyzed using FloJo v10 (FloJo, LLC). Live cells were gated and from these, CD45+ cells selected. Of these, CD8+ T cells (CD3+CD8+), effector CD8+ T cells (CD3+CD8+CD44hiCD62Llo), CD4+ T cells (CD3+CD4+), NK cells (CD3-CD49b+), neutrophils $(\mathrm{Cd} 11 \mathrm{~b}+\mathrm{Gr} 1+)$ and macrophages $(\mathrm{CD} 11 \mathrm{~b}+\mathrm{F} 4 / 80+)$ were identified.

\section{In vivo studies}

All in vivo studies were carried out under the terms of the Home Office Project Licence PPL 70/6030 and subject to Queen Mary University of London ethical review, according to the guidelines for the welfare and use of animals in cancer research. $1 \times 10^{6}$ LLC or LY2 cells or $3 \times 10^{6}$ DT6606 cells were injected subcutaneously into the right flank of 6 -week-old C57/BL6 mice. $1 \times 10^{5} 4 \mathrm{~T} 1$ cells were injected into the mammary fat pads of female $\mathrm{BALB} / \mathrm{c}$ mice to establish orthotopic tumors. $2 \times 10^{6} \mathrm{CT} 26$ cells were injected subcutaneously into the right flank of 6-week-old Balb/C mice. $2 \times 10^{6} \mathrm{SCCVII}$ cells were injected subcutaneously into the right flank of 6-week-old $\mathrm{C} 3 \mathrm{H} /$ HeN mice. $1 \times 10^{7}$ HCPC1 cells were injected subcutaneously into the right flank of 4-week-old Syrian hamsters. When tumors were palpable, they were stratified into treatment groups and received i.t. injections of $1 \times 10^{8} \mathrm{PFU}$ of virus or vehicle buffer in a total volume of $50 \mu \mathrm{L}$. Tumor growth was measured using electronic callipers until tumors reached a volume of $1.44 \mathrm{~cm}^{3}$ at which point the animals were sacrified, and the area calculated according to the following formula:

$$
\text { Tumor Volume }=\frac{\pi w^{2} l}{6}
$$

where $\mathrm{w}$ is width and $\mathrm{l}$ is length. 
Tumor growth curves were terminated on the death of the first animal in each group, but group survival was monitored until the experimental endpoint and KaplanMeier survival plots generated.

In the neoadjuvant surgical models, tumors were excised under continuous isofluorane and nitrous oxide via nose cone and wounds closed with interrupted 4-0 monocryl absorbable sutures (Ethicon, Livingston, UK). To capture regrowth from microscopic as opposed to macroscopic residual disease, any tumor that clinically regrew within a week of the operation were excluded. Mice were followed up via twice-weekly weight measurements and assessment of general well-being until visibly unwell or a reduction in a maximum of $20 \%$ of body weight was noted.

\section{Immune cell depletion}

The VV $\Delta$ TK $\triangle$ N1L treatment arm of the LLC surgical neoadjuvant experiment was repeated after depletion of NK, CD4+ or CD8+ cells. One day prior to commencement of i.t. viral treatment, $200 \mu \mathrm{g}$ of rat anti-CD4 IgG (clone GK1.5), anti-CD8 IgG (clone TIB210), anti-NK IgG (clone PK136) or control IgG in $200 \mu \mathrm{L}$ PBS was injected intraperitoneally. Injections were continued twice weekly for the duration of the experiment. Depletion was verified using flow cytometric assessment of splenocytes 48 hours post administration.

\section{Preparation of tumor tissue}

Subcutaneous tumors were diced and incubated in collagenase D $(1 \mathrm{mg} / \mathrm{mL}$; Roche $)$ and DNAse I $(0.1 \mathrm{mg} / \mathrm{mL}$; Roche) in PBS, at $37^{\circ} \mathrm{C}$ for 2 hours. Homogenates were filtered through $70 \mu \mathrm{m}$ cell strainers and resuspended in FB.

For cytokine screening, harvested tumors were homogenized in a 10-fold dilution ( $\mathrm{w} / \mathrm{v}$ ) of tissue extraction buffer (prepared by dissolving one complete ULTRA minitablet (Roche) into $10 \mathrm{~mL}$ of $50 \mathrm{mM}$ Tris- $\mathrm{HCl}, \mathrm{pH}$ 7.4). Following high-speed centrifugation, the total protein concentration $(\mathrm{g} / \mathrm{mL})$ of supernatant samples was obtained using a NanoDrop 1000 Spectrophotometer and subsequently used to normalize multianalyte cytokine/chemokine values.

\section{Preparation of splenocytes}

Harvested spleens were flushed through $70 \mu \mathrm{m}$ BD Falcon cell strainers with complete T-cell media (RPMI medium 1640 (Sigma Aldrich), 10\% FCS, 1\% streptomycin/penicillin, $1 \%$ sodium pyruvate and $1 \%$ non-essential amino acids (Gibco) and 0.1\% $\beta$-mercaptoethanol). Red blood cells (RBCs) were lysed using RBC lysis buffer (SigmaAldrich) and resuspended in complete T-cell medium.

\section{Preparation of blood leukocytes}

Blood was extracted via hepatic vein cannulation and samples were added to a heparinized microcentrifuge tube, resuspended in $1 \mathrm{~mL}$ of RBC lysis buffer and filtered through $70 \mu \mathrm{m}$ cell strainers. Single cells were resuspended in FB.

\section{Macrophage and dendritic cell (DC) culture}

Red marrow was flushed out from femurs and tibias of C57Bl/6 mice using RPMI medium. Cells were homogenized with a 23G needle and syringe, filtered through $70 \mu \mathrm{m}$ cell strainers and resuspended in RBC lysis buffer followed by resuspension in complete $\mathrm{T}$ cell medium containing either M-CSF or GMCSF (Roche) at $30 \mathrm{ng} /$ $\mathrm{mL}$ to selectively enrich and grow macrophages or DCs, respectively. At day 7 , adherent macrophages were washed with PBS, gently scraped from the plates and resuspended in growth factor-free complete medium. Non-adherent DCs were used at day 7. Purity was confirmed by staining cells with fluorophore-labelled antibodies specifying DCs (CD11c+MHCII+) and monocyte (CD11b+F4/80+) populations. Enriched populations were validated using FACS and infected with the indicted viruses for 24 hours at an MOI of $1 \mathrm{PFU} /$ cell. Infection was confirmed visually by fluorescent microscope observation of virally encoded RFP expression.

\section{Assessment of lung and lymph node metastases}

Flank LLC tumor grafts were monitored via calliper measurement until a treatment group reached the sacrificial end point. All animals were euthanized at the same time; lungs harvested and any gross tumor deposits noted. Lung lobes were separated, fixed in $4 \%$ formaldehyde, paraffin embedded and cut into $4 \mu \mathrm{m}$ sections with a Leica EG1160 microtome (Leica Microsystems UK, Milton Keynes, UK). They were subsequently stained with H\&E. For each lobe, slices were also performed above and below the largest cross section. All three sections were scrutinized for tumor deposits via light microscopy by an experienced pathologist who was blinded to treatment groups.

\section{Statistical analysis}

Graph Pad Prism 6 was used for comparative statistical analysis. Dual condition comparisons or normally distributed data were made using the unpaired Student's t-test. For more than one condition, one or two-way analysis of variances (ANOVAs), respectively, were performed, with post-hoc Tukey tests to compare treatment pairs. Where data were categorical, specific treatment pairs were compared with Fisher's exact tests following subclassification into multiple $2 \times 2$ tables. Survival data were represented by Kaplan-Meier plots with log rank analyses to delineate whether any differences between specific treatment pairs were statistically significant.

\section{RESULTS \\ VV $\Delta T K \Delta N 1 L$ is an effective primary antitumor therapeutic in murine models of pancreatic cancer}

The TK-deleted VV, VVL15, was manipulated as described in the methods to interrupt the N1L region, replacing it with an RFP marker under control of the endogenous H5 promoter, creating VV $\Delta \mathrm{TK} \Delta \mathrm{N} 1 \mathrm{~L}$. The deletion of N1L did not negate cytotoxicity compared with VVL15 
$(\mathrm{VV} \Delta \mathrm{TK})$ in all murine and human tumor cell lines examined (online supplementary figure S1A) and the virus remained replication competent. Replication was modestly, but significantly attenuated in many cell lines, which was unsurprising given that viral replication has been inversely correlated to the extent to which it has been manipulated (online supplementary figure S1B). In vivo, $\mathrm{VV} \Delta \mathrm{TK} \Delta \mathrm{N} 1 \mathrm{~L}$ was able to exert significantly more control over murine pancreatic DT6606 subcutaneous tumor growth compared with VVATK, which translated into substantially improved survival in this model (figure 1A,B). This effect was also evidence, although far less pronounces in a CT26 model of colorectal cancer (figure 1C,D) and more aggressive SCCVII squamous cell carcinoma (figure 1E,F) and LLC metastatic lung cancer (figure 1G,H) flank models, in which both viruses were only able to exert limited control over tumor growth, although $\mathrm{VV} \Delta \mathrm{TK} \Delta \mathrm{N} 1 \mathrm{~L}$ was able to extend survival significantly compared with PBS treatment in each model.

\section{VV $\Delta T K \Delta N 1 L$ induces adaptive antitumor immune responses to mediate efficacy in murine models of pancreatic cancer}

Given the ability of VVATK $\Delta$ N1L to control DT6606 murine pancreatic tumors, we investigated the functional mechanisms responsible for extended survival in this model. Ex vivo restimulation of splenocytes with growtharrested tumor cells demonstrated a significant enhancement of tumor-specific IFN $\gamma$ production up to 2 weeks after mice were treated with a single dose of VV $\Delta \mathrm{TK} \Delta \mathrm{N} 1 \mathrm{~L}$ (figure 2A), an effect also evident when splenocytes were restimulated ex vivo with a peptide representing the mesothelin epitope, overexpressed in pancreatic cancer (figure 2B).

Cytotoxic $\mathrm{T}$ cell assays confirmed the superior ability of splenocytes from VV $\Delta \mathrm{TK} \Delta \mathrm{N} 1 \mathrm{~L}$-treated animals to specifically lyse target tumor cells, but not unrelated cells, ex vivo (online supplementary figure S2A). FACS analysis of splenocytes at days 7 and 14 post injection indicated that VV $\Delta \mathrm{TK} \Delta \mathrm{N} 1 \mathrm{~L}$ treatment enhanced effector $\mathrm{CD} 8+$ populations (figure 2C) and increased the number of CD8+ and CD4+ $\mathrm{T}$ cells infiltrating into tumors (figure 2D), demonstrating an effective impact on generation of antitumor adaptive immune responses in vivo. In vitro, VVL recombinants were able to enhance activation of both bone marrow-derived DCs (figure 2E) and macrophages (figure $2 \mathrm{~F}$ ) and VV $\Delta \mathrm{TK} \Delta \mathrm{N} 1 \mathrm{~L}$ treatment resulted in a shift of the macrophage pool into professional APCs as evidenced by the significant upregulation of MHCII 24 hours post infection, suggesting a phenotypic switch of macrophages to M1-polarized antitumor populations. To further confirm the requirement for adaptive immunity to mediate the observed therapeutic effect, immune subset depletion assays were carried out that demonstrated primary antitumor efficacy could be maintained when CD4+ Tand NK cells were depleted, but CD8+ T cells were absolutely required to deliver a therapeutic effect after treatment (figure 2G). These data suggest that the therapeutic efficacy of VV $\Delta \mathrm{TK} \Delta \mathrm{N} 1 \mathrm{~L}$ is conferred by enhanced APC and $\mathrm{T}$ cell activation and intratumoral infiltration in the murine pancreatic model.

\section{VV $\Delta T K \Delta N 1 L$ decreases metastasis to extend survival in an aggressive murine model of metastatic lung cancer}

VV $\Delta \mathrm{TK} \Delta \mathrm{N} 1 \mathrm{~L}$ was not effective at controlling primary tumor burden in more aggressive tumor models, but it was noted that in the murine lung cancer model survival was significantly, but modestly, extended after treatment compared with treatment using VV $\Delta \mathrm{TK}$. Analysis of lung metastasis in this model suggested that this survival effect was associated with superior control of metastatic spread of the primary tumor (online supplementary figure 3A, S2B). Ex vivo restimulation of splenocytes with growtharrested tumor cells again demonstrated a significant enhancement of tumor-specific IFN $\gamma$ production up to 2 weeks after mice were treated with a single dose of VV $\Delta \mathrm{T}$ $\mathrm{K} \Delta \mathrm{N} 1 \mathrm{~L}$ (figure 3B), increased activation and cytotoxicity of CD8+ T cells (figure 3C; online supplementary figure S2C) and increased infiltration of CD4+ and CD8+ T cells into the tumor (figure 3D). However, the extent to which $\mathrm{VV} \Delta \mathrm{TK} \Delta \mathrm{N} 1 \mathrm{~L}$ was able to activate and elevate $\mathrm{T}$ cells was low in comparison to those seen using the DT6606 pancreatic cancer model. The LLC model of lung cancer is an extremely aggressive subcutaneous model and although $\mathrm{VV} \Delta \mathrm{TK} \Delta \mathrm{N} 1 \mathrm{~L}$ was able to enhance $\mathrm{T}$ cell infiltration into the tumor and generate effective antitumor effector $\mathrm{T}$ cell responses, the time needed to generate these responses and the comparatively weak responses generated is likely to be too weak and slow to prevent demise of the animal by tumor dissemination.

As VV $\Delta \mathrm{TK} \Delta \mathrm{N} 1 \mathrm{~L}$ was able to limit metastasis in this model to a significantly greater extent than VV $\Delta \mathrm{TK}$ treatment, we investigated early induction of innate immune responses as a potential explanation for metastatic control. Following a single i.t. dose of virus into LLC tumor-bearing mice, both viruses were able to enhance neutrophil infiltration into the tumor, with VV $\Delta \mathrm{T}$ $\mathrm{K} \Delta \mathrm{N} 1 \mathrm{~L}$ having a superior effect compared with VV $\Delta \mathrm{TK}$ (figure 3E). Conversely, macrophage populations within the tumor were significantly reduced by viral treatment (figure 3F,G). These results were mirrored in similar experiments investigating neutrophil and macrophage accumulation in DT6606 tumors (online supplementary figure S2D,E). i.t. NK cells were undetectable; however, VV $\triangle \mathrm{TK} \Delta \mathrm{N} 1 \mathrm{~L}$ treatment was able to induce a significant increase in circulating NK cells, critical for control of tumor dissemination from the primary tumor, at early time points after injection into LLC tumors (figure $3 \mathrm{H}$ ). These results suggest that in aggressive tumor models, the adaptive immune environment programmed by $\mathrm{VV} \Delta \mathrm{TK} \Delta \mathrm{N} 1 \mathrm{~L}$ is insufficient to control the rapidly developing primary tumor, but the innate immunity changes conferred by treatment are able to reduce metastatic spread from the primary tumor, extending mortality in treated animals. 


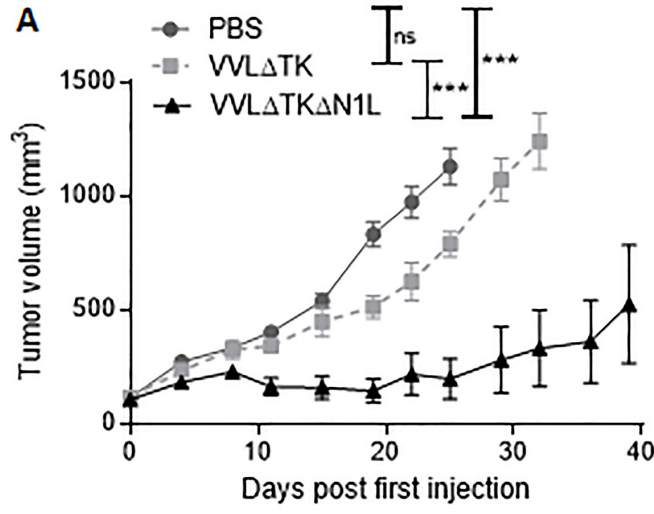

C

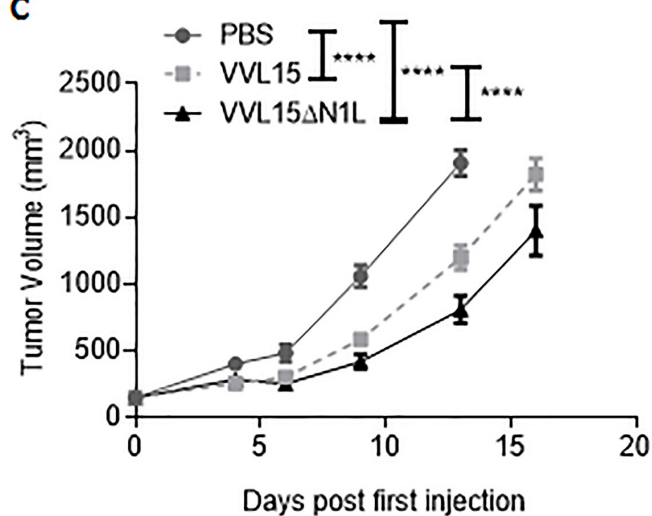

$\mathbf{E}$

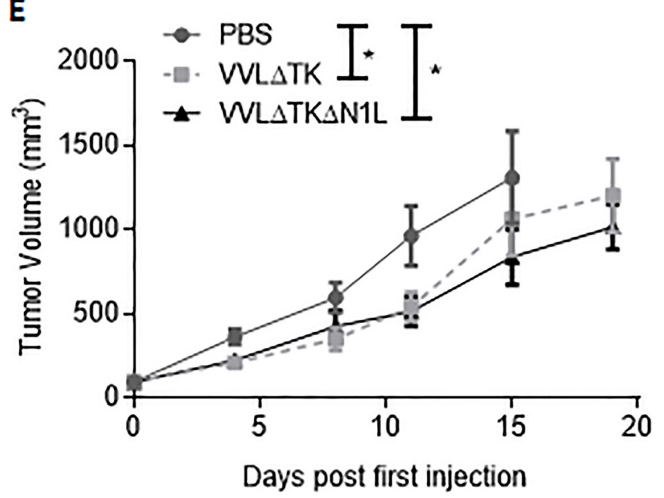

G

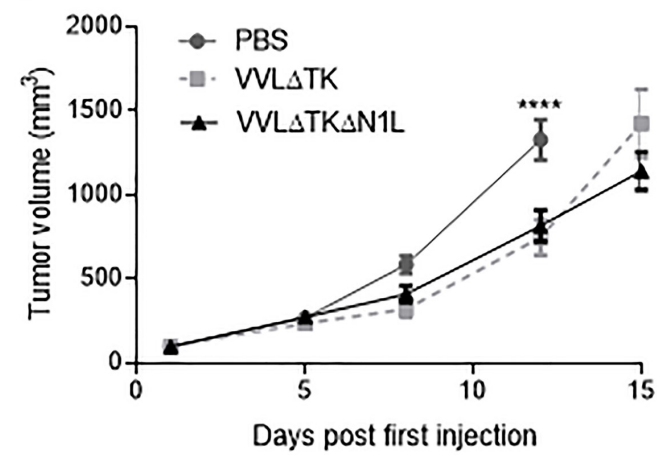

B

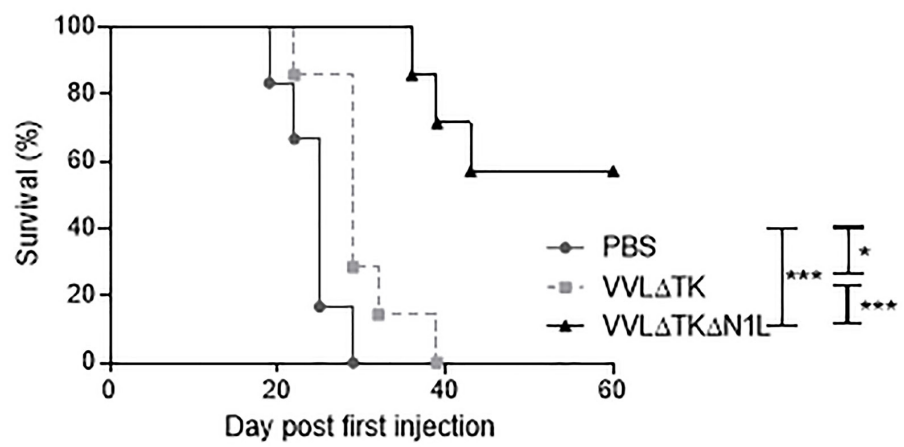

D
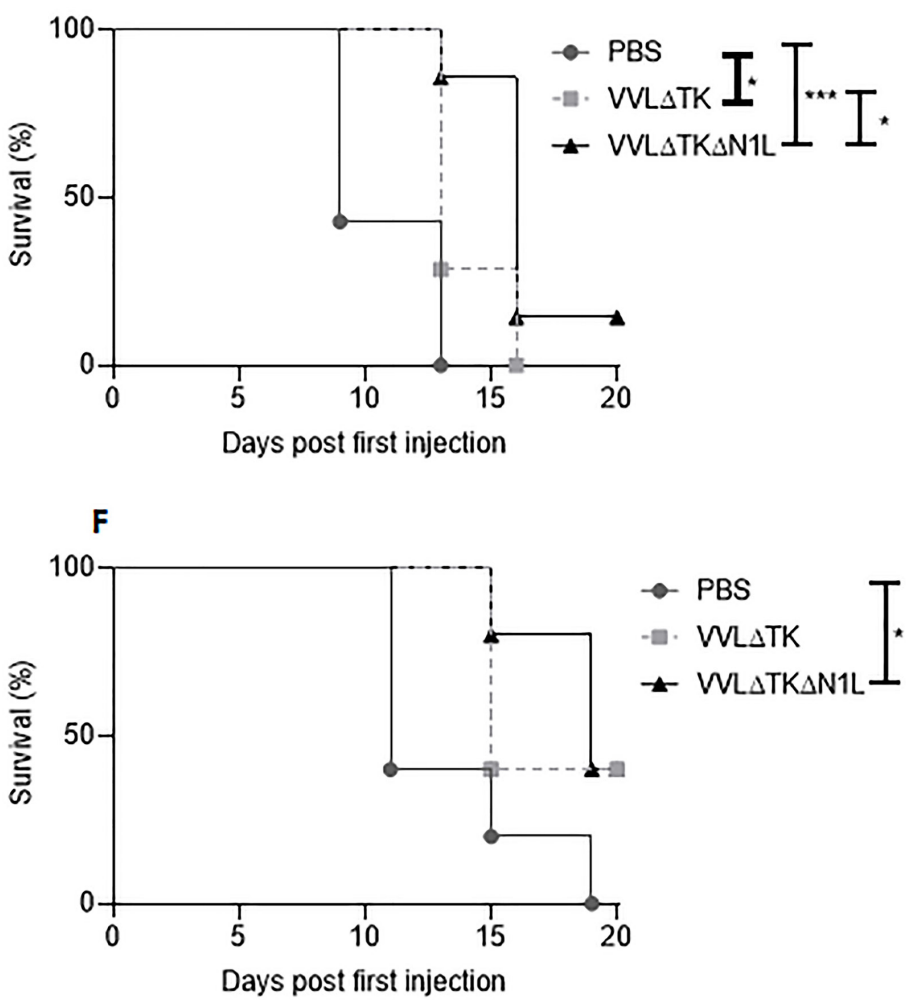

H

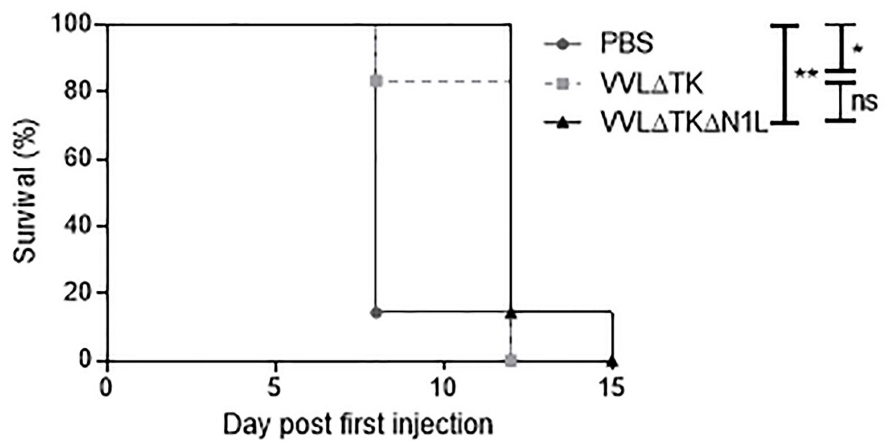

Figure 1 VV $\triangle T K \Delta N 1 L$ demonstrates antitumor efficacy in vivo. (A) and (B) DT6606 pancreatic tumors, (C) and (D) CT26 colorectal tumors, (E) and (F) SCCVII oral cavity squamous carcinoma and $(\mathrm{G})$ and $(\mathrm{H})$ Lewis lung carcinoma were established in the flanks of immune-competent mice ( $n=5-7 /$ group). Once palpable, mice were injected intratumorally daily for 5 days with $1 \times 10^{8}$ PFU VV $\triangle T K, V V \Delta T K \Delta N 1 L$ or PBS. (A), (C), (E), and (G) Tumor growth was monitored and a two-way analysis of variance with post hoc Tukey tests used to compare significance at all time points. Significance at (A) day 25 or (B)-(G) day 20 is shown. (B), (D), (F), and (H) Kaplan-Meier survival analysis with Log rank (Mantel-Cox) tests was used to assess survival. In all cases, the mean \pm SEM is shown. ${ }^{*} \mathrm{p}<0.05 ;{ }^{* *} \mathrm{p}<0.01 ;{ }^{* \star *} \mathrm{p}<0.001 ;{ }^{* * \star} \mathrm{p}<0.0001$. 
A

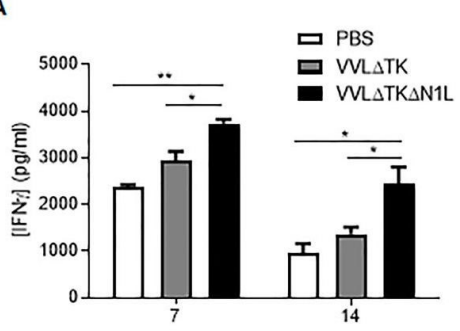

Day post virus injection

C

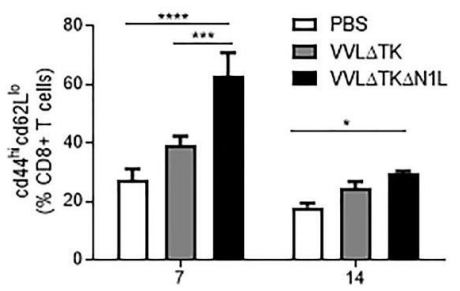

Day post virus injection

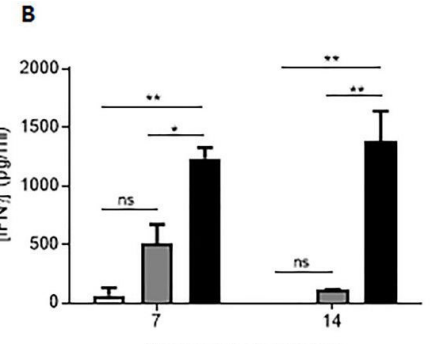

Day post virus injection
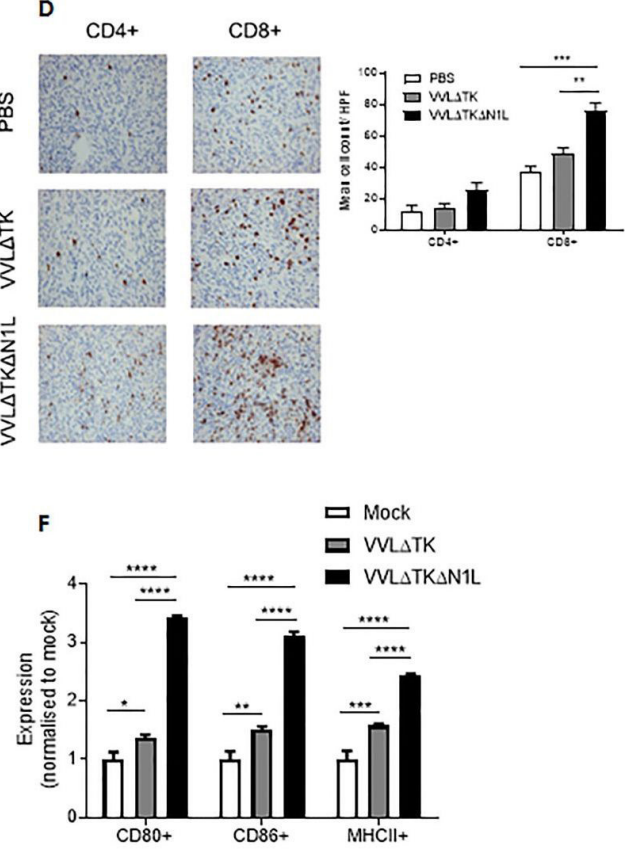

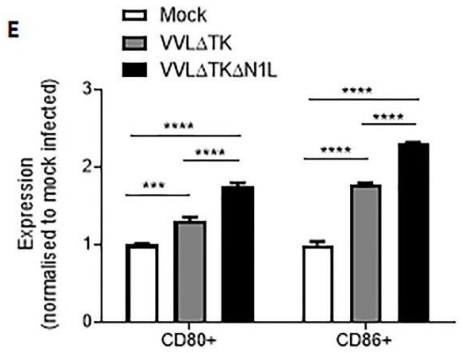

G

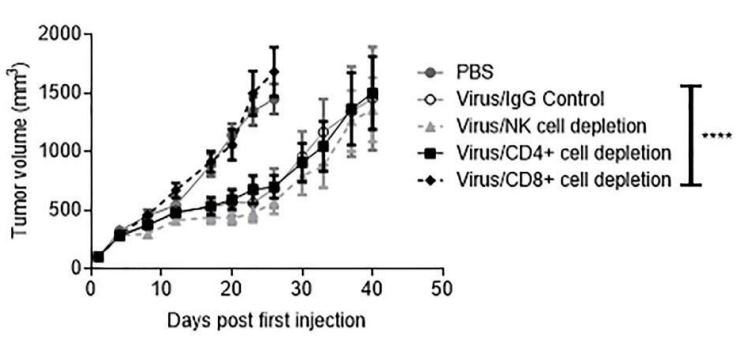

Figure 2 VV $\triangle T K \Delta N 1 L$ induces adaptive immune responses against pancreatic cancer in vivo. DT6606 tumors were established in the flanks of immune-competent C57/BI6 mice ( $n=3-4 / g r o u p)$. Once palpable, mice were injected intratumorally (i.t) once with $1 \times 10^{8}$ PFU VV $\triangle T K$, VV $\triangle T K \triangle N 1 L$ or PBS. Splenocytes were collected 7 or 14 days after treatment and analyzed. (A) Splenocytes were analyzed ex vivo for response to growth-arrested DT6606 cells using coculture for 72 hours followed by interferon $\gamma($ IFN $\gamma$ ) ELISA. (B) Splenocytes were analyzed ex vivo for response to mesothelin peptide using IFN $\gamma$ ELISA after a 72-hour stimulation. (C) Splenocytes were collected and analyzed using FACS for expression of CD45, CD3, CD8, CD44 and CD62L. Percentage CD44 ${ }^{\text {Ci }}$ CD62L $^{\text {lo }}$ cells (of live, CD45+/CD3+/CD8+ cells) are shown. (D) Tumors were collected 14 days post treatment and stained for CD4+ or CD8+ cells. Representative immunohistochemistry (IHC) images are shown (original magnification x200) and cells/higher power field (HPF) shown graphically after 15 HPFs were counted. Of note, CD4+ staining cannot exclude the presence of TReg cells within the tumor. (E) and (F) Dendritic cells (DCs) and macrophages were matured from bone marrow precursors taken from C57/BI6 mice using granulocyte-macrophage colony-stimulating factor and macrophage colony-stimulating factor, respectively. Enriched populations were validated using FACS and infected with the indicted viruses for 24 hours at an MOI of 1 PFU/cell ( $n=3 / g r o u p)$. (E) Expression of CD80 and CD86 markers on enriched DCs was examined after in vitro infection using FACS and normalized to expression levels on mock (PBS)-infected cells. (F) Expression of CD80, CD86 and major histocompatibility complex II (MHCII) markers on enriched macrophages was examined after infection using FACS and normalized to expression levels on mock (PBS)-infected cells. In all cases, one-way analysis of variance (ANOVA) with post hoc Tukey tests was used to assess significance at each time point. (G) Cell depleting or lgG control antibodies were commenced intraperitoneally in mice bearing DT6606 flank tumors a day prior to the first i.t treatment of virus ( $n=7 /$ group). Mice were treated as above and tumor growth monitored. A two-way ANOVA with post hoc Tukey tests was used to assess significance. In all cases, the mean \pm SEM is shown. ${ }^{*} \mathrm{p}<0.05 ;{ }^{* \star} \mathrm{p}<0.01 ;{ }^{* \star *} \mathrm{p}<0.001 ;{ }^{* \star \star} \mathrm{p}<0.0001$. 
A

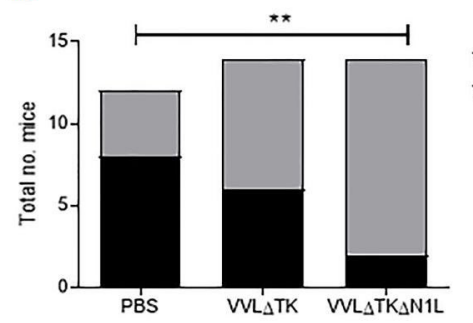

C

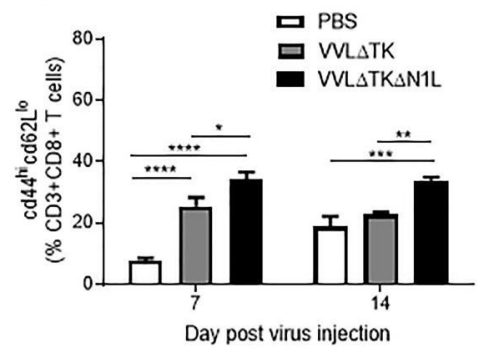

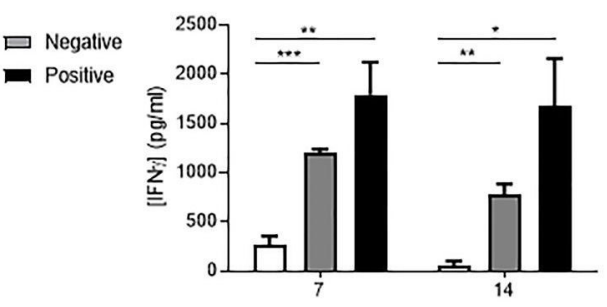

Day post virus injection

D

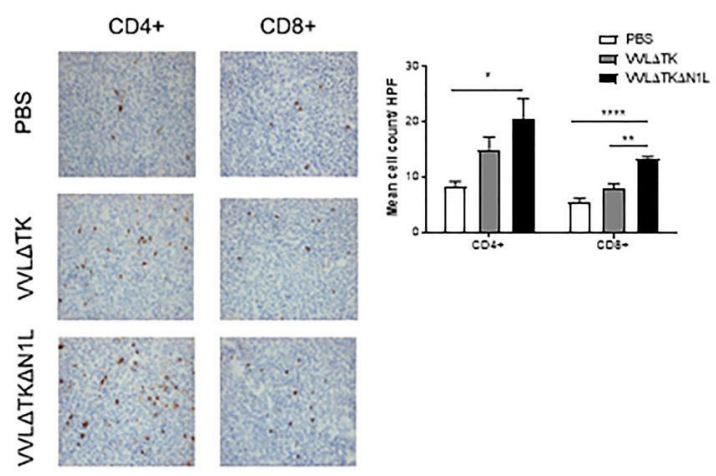

$\mathbf{F}$
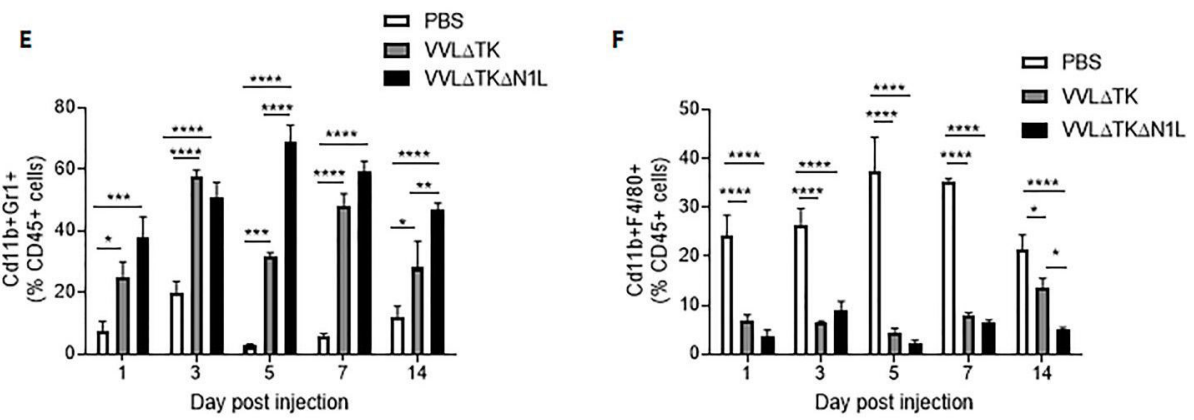

G

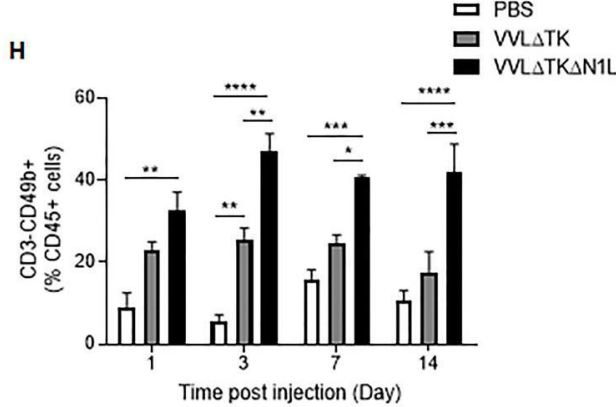

Figure 3 VV $\triangle T K \Delta N 1 L$ induces adaptive and innate immunity against lung cancer in vivo. Lewis lung carcinoma (LLC) tumors were established in the flanks of immune-competent C57/BI6 mice. (A) Once palpable, mice were injected intratumorally (i.t) daily for 5 days with $1 \times 10^{8} \mathrm{PFU}$ VV $\triangle T K, \mathrm{VV} \Delta T K \Delta N 1 \mathrm{~L}$ or PBS ( $\mathrm{n}=14 /$ group). Mice were sacrificed at day 15 , lungs H\&E stained and analyzed for the presence of metastasis by a pathologist blinded to treatment groups. Lungs were scored as negative or positive and a Fishers exact test used to determine significance. $(B)-(H)$ Once palpable, mice were injected i.t once with $1 \times 10^{8} \mathrm{PFU}$ VV $\triangle T K$, VV $\triangle T K \Delta N 1 \mathrm{~L}$ or PBS (n=3-4/group). (B) Splenocytes were analyzed ex vivo for response to growth-arrested LLC cells using coculture for 72 hours followed by interferon $\gamma$ (IFN $\gamma$ ) ELISA. (C) Splenocytes were collected and analyzed using FACS for expression of CD45, CD3, CD8, CD44 and CD62L. Percentage CD44 hi CD62L lo cells (of live, CD45+/CD3+/CD8+ cells) are shown. (D) Tumors were collected 14 days post treatment and stained for CD4+ or CD8+ cells. Representative IHC images are shown (magnification $\times 200$ ) and cells/HPF shown graphically after 15 HPFs were counted. Of note, CD4+ staining cannot exclude the presence of TReg cells within the tumor. (E) FACS analysis was used to assess CD11b+Gr1+ neutrophils in tumors at the indicated time points. (F) FACS analysis was used to assess CD11b+F4/80+ macrophages in the tumors at the indicated time points. (G) Fourteen days post infection tumor sections were stained with $\mathrm{F} 4 / 80$ to assess macrophage infiltration. Representative images are shown. Magnification x200. (H) FACS analysis was used to assess CD3-CD49b+ natural killer cells in blood at the indicated time points. In all cases, one-way analysis of variance with post hoc Tukey tests was used to assess significance at each time point. In all cases, the mean \pm SEM is shown. ${ }^{*} \mathrm{p}<0.05 ;{ }^{* *} \mathrm{p}<0.01 ;{ }^{* * *} \mathrm{p}<0.001 ;{ }^{* * *} \mathrm{p}<0.0001$. 
VV $\Delta T K \Delta N 1 L$ can perturb the biochemical microenvironment of the tumor in an aggressive murine lung cancer model

To delineate how VV $\Delta \mathrm{TK} \Delta \mathrm{N} 1 \mathrm{~L}$ was able to effect early innate cellular changes, syngeneic LLC flank tumors were collected after one i.t. dose of virus and screened for common inflammatory cytokines and chemokines via multianalyte ELISAs. IL- $\alpha$, IL1- $\beta$ and GCSF cytokines were consistently significantly elevated after VV $\Delta \mathrm{TK} \Delta \mathrm{N} 1 \mathrm{~L}$ treatment (figure 4A; online supplementary figure S3A-C), likewise for the chemokines MIP-1 $\alpha$ and KC (figure 4B; online supplementary figure S3D-E). Interestingly, IL-12, a major DC-secreted mediator of NK effector function, was not detected in tumors infected with either virus.

Investigations into the sources of elevated immune mediators revealed that the murine chemokine KC (CXCL1), a functional homologue of human IL-8 important for neutrophil recruitment, was produced at much higher levels following infection of LLC tumor cells in comparison to DCs or monocytes (figure 4C). Infection with VVATKAN1L enhanced production of KC in comparison to VVATK across all cell lines, although uninfected LLC tumor cells produced significant basal levels of KC. GCSF aids the recruitment and maturation of neutrophils and other myeloid cells and its production from infected DCs and monocytes occurred almost universally following $\mathrm{VV} \Delta \mathrm{TK} \Delta \mathrm{N} 1 \mathrm{~L}$ infection (figure 4D). MIP1 $\alpha$, a chemokine facilitating the recruitment and activation of myeloidderived cells, NK cell migration and the generation of a CD8+ cytotoxic T lymphocyte (CTL) memory responses ${ }^{21}$ were also secreted from infected DCs and macrophages and VV $\Delta$ TK $\triangle$ N1L infection markedly increased output from both these cell populations in vitro (figure $4 \mathrm{E}$ ). The classic acute phase reactant cytokines, IL1- $\alpha$ and IL1- $\beta$, play a major role in the recruitment and activation of macrophages and neutrophils as well as lymphocytes. Further, IL1- $\beta$ may help drive a Th-1 type antigen-specific CTL response. ${ }^{22}$ Both these cytokines were secreted from DCs and macrophages almost exclusively in response to $\mathrm{VV} \Delta \mathrm{TK} \Delta \mathrm{N} 1 \mathrm{~L}$ (figure $4 \mathrm{~F}, \mathrm{G}$ ). In addition, VV $\Delta \mathrm{TK} \Delta \mathrm{N} 1 \mathrm{~L}$ was also able to significantly enhance IL-18 secretion from DCs and macrophages in vitro (figure 4H). IL-I $\beta$ and IL-18 act as surrogate markers for intracellular activation of inflammasome platforms, suggesting that the VV N1L protein may additionally downregulate inflammasome signaling. Thus, it appears that by releasing suppression on the NFKB pathway, VV $\Delta \mathrm{TK} \Delta \mathrm{N} 1 \mathrm{~L}$ can dramatically alter the levels of inflammatory mediators expressed within the TME, the major source of which appear to be macrophages and DCs. These changes can explain the profound effects on innate NK and neutrophil populations and also the elevation in $\mathrm{T}$ cell responses seen as a result of OV treatment.

\section{VV $\Delta T K \Delta N 1 L$ can enhance postoperative survival in aggressive} metastatic tumor models

LLC tumors avidly infiltrate underlying tissue and readily metastasize to the lungs; thus, surgical excision is likely to leave foci of residual disease, which has been

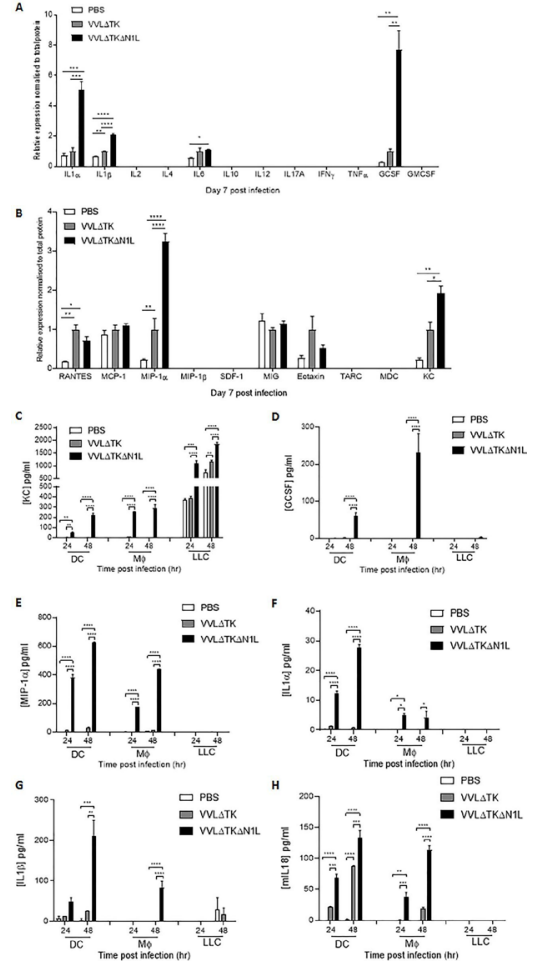

Figure 4 VV $\triangle T K \Delta N 1 \mathrm{~L}$ alters antigen-presenting cell secretion of immune modulators. $(A)$ and $(B)$ Lewis lung carcinoma (LLC) tumors were established in the flanks of immune-competent C57/BI6 mice ( $n=3-4 /$ group). Once palpable, mice were injected intratumorally once with $1 \times 10^{8} \mathrm{PFU}$ VV $\triangle \mathrm{TK}, \mathrm{V} \triangle \mathrm{TK} \triangle \mathrm{N} 1 \mathrm{~L}$ or PBS. One-way analysis of variance (ANOVA) with post hoc Tukey tests was used to assess significance. (A) At 7 days post infection, tumors were harvested and homogenized and their supernatant analyzed for the presence of 12 common inflammatory cytokines by multianalyte ELISA. Relative expression was normalized to total protein. (B) At 7 days post infection, tumors were harvested and homogenized and their supernatant analyzed for the presence of 12 common inflammatory chemokines by multianalyte ELISA. Relative expression was normalized to total protein. $(\mathrm{C})-(\mathrm{H})$ Dendritic cell (DC) and macrophage populations were matured from bone marrow as previously and infected with virus at an $\mathrm{MOI}$ of $1 \mathrm{PFU} /$ cell for 24 and 48 hours. LLC cells were infected and analyzed in parallel. (C) Supernatants were analyzed for the presence of keratinocyte chemoattractant (KC) using ELISA. (D) Supernatants were analyzed for the presence of granulocyte colony-stimulating factor (GCSF) using ELISA. (E) Supernatants were analyzed for the presence of macrophage inflammatory protein (MIP)$1 \alpha$ using ELISA. (F) Supernatants were analyzed for the presence of interleukin (IL)-1 $\alpha$ using ELISA. (G) Supernatants were analyzed for the presence of IL-1 $\beta$ using ELISA. $(H)$ Supernatants were analyzed for the presence of IL-18 using ELISA. Two-way ANOVA with post hoc Tukey tests was used to assess significance. In all cases, the mean \pm SEM is shown. ${ }^{*} p<0.05 ;{ }^{* *} p<0.01 ;{ }^{* * *} p<0.001 ;{ }^{* * * *} p<0.0001$. GMCSF, granulocyte-macrophage colony-stimulating factor; IFN $\gamma$, interferon $\gamma$. RANTES; regulated on activation, normal $\mathrm{T}$ cell expressed and secreted, TNFa; tumor necrosis factor a, MCP-1; Monocyte Chemoattractant Protein-1, SDF-1; stromal cell-derived factor 1, MIG; monokine induced by gamma interferon, TARC; Thymus- And Activation-Regulated Chemokine, MDC; Macrophage-derived Chemokine. 
A
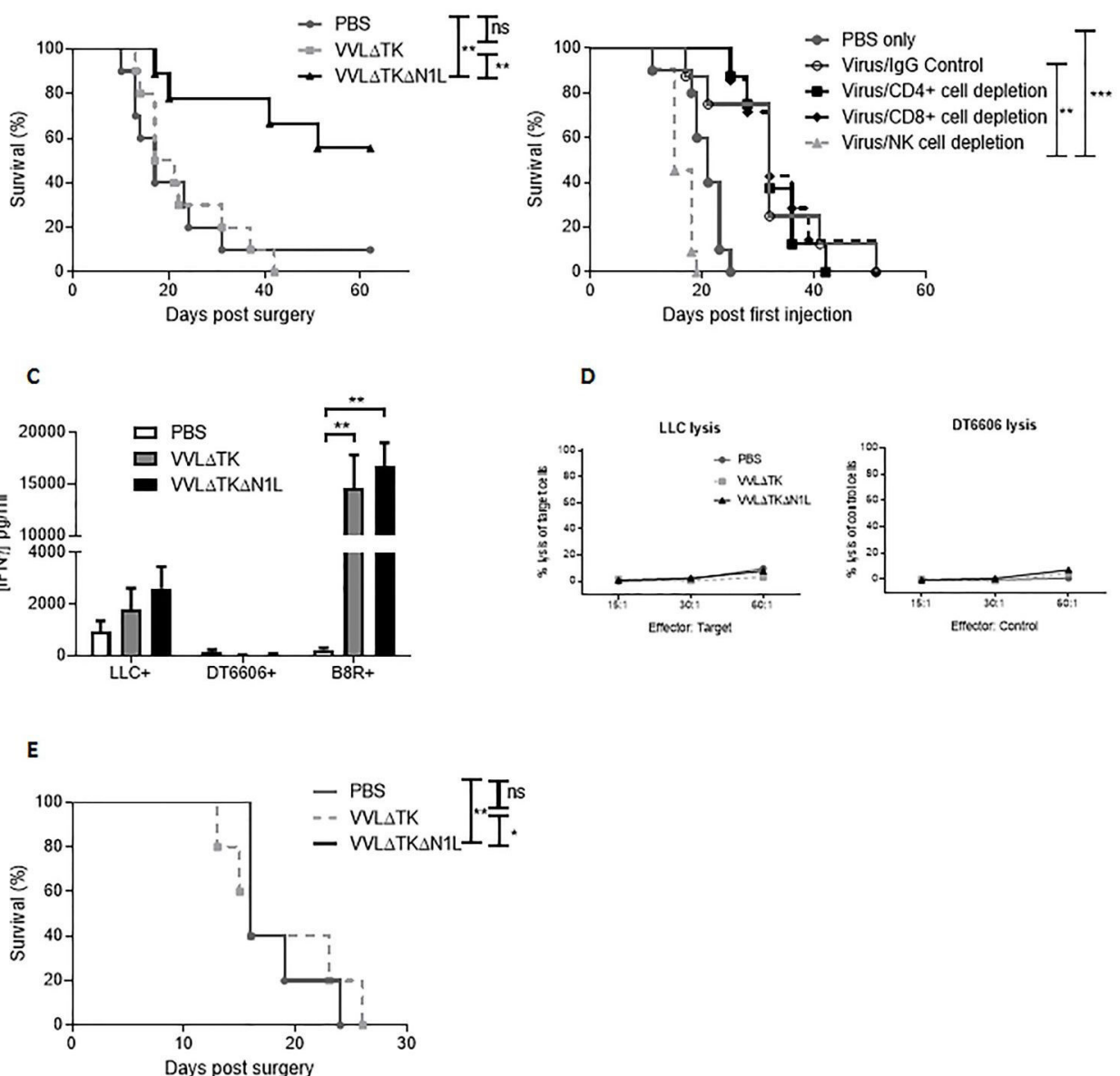

Figure 5 VV $\triangle T K \triangle N 1 L$ is an effective surgical adjuvant in vivo. (A) Lewis lung carcinoma (LLC) tumors were established in the flanks of immune-competent C57/BI6 mice ( $\mathrm{n}=7-10 /$ group). Once palpable, mice were injected intratumoral (i.t) with $1 \times 10^{8} \mathrm{PFU}$ $\mathrm{VV} \triangle \mathrm{TK}, \mathrm{V} \triangle \mathrm{TK} \triangle \mathrm{N} 1 \mathrm{~L}$ or PBS daily for 5 days. Tumors were surgically resected 7 days after the final virus dose. Kaplan-Meier survival analysis with log rank (Mantel-Cox) tests was used to assess survival. (B) Cell depleting or lgG control antibodies were commenced intraperitoneally in mice bearing LLC flank tumors a day prior to the first i.t treatment of virus ( $n=7 / g r o u p)$. Mice were treated and resected as in (A) and Kaplan-Meier survival analysis with log rank (Mantel-Cox) tests was used to assess survival. (C) Mice were treated as in (A) and 7 days post resection, splenocytes were analyzed for response to growth-arrested LLC or DT6606 (control) tumor cells ex vivo using interferon $\gamma$ (IFN $\gamma$ ) ELISA after 72 hours coculture ( $n=3-4 / g r o u p)$. Response of the splenocytes to the Vaccinia virus B8R peptide was assessed in parallel. One-way analysis of variance with post hoc Tukey tests was used to assess significance. (D) Splenocytes isolated as in (C) were cocultured with growth-arrested tumor cells for 5 days, pooled and further cultured with 5000 target (LLC) or control (DT6606) tumor cells at 15:1, 30:1 and 60:1 ratios. Non-radioactive Lactate dehydrogenase $(\mathrm{LDH})$ release assays were performed to determine percentage tumor cell lysis. (E) Orthotopic 4T1 breast tumors were established in immune-competent Balb/C mice ( $\mathrm{n}=5 / \mathrm{group})$ and treated with three doses of virus at $1 \times 10^{8}$ PFU. Five days later, tumors were surgically resected. Kaplan-Meier survival analysis with log rank (Mantel-Cox) tests was used to assess survival. In all cases, the mean \pm SEM is shown. ${ }^{\star} \mathrm{p}<0.05 ;{ }^{\star *} \mathrm{p}<0.01$; ${ }^{\star \star *} \mathrm{p}<0.001$. NK, natural killer.

well characterized as an ideal model of MRD postsurgical resection. ${ }^{23}$ Given the suppressive effects of the N1L protein on NK populations and the involvement in NK cells in control of tumor dissemination, we next explored the use of VVDTK $\Delta \mathrm{N} 1 \mathrm{~L}$ as a neoadjuvant alongside surgical excision of tumors, a scenario which may better realize the curative potential of $\mathrm{OV}$ therapy. Twelve days prior to surgical excision, PBS, VVATK or VVATK $\Delta$ N1L were administered i.t. daily for 5 days. There was a significant postoperative survival advantage favoring neoadjuvant i.t. VV $\Delta \mathrm{TK} \Delta \mathrm{N} 1 \mathrm{~L}$ treatment (figure 5A). Given the effects of $\mathrm{VV} \Delta \mathrm{TK} \Delta \mathrm{N} 1 \mathrm{~L}$ on systemic $\mathrm{T}$ and $\mathrm{NK}$ populations, the $\mathrm{VV} \Delta \mathrm{TK} \Delta \mathrm{N} 1 \mathrm{~L}$ neoadjuvant therapy regime was repeated in LLC flank tumor-bearing mice in which NK, CD4+ or CD8+ T cells were selectively depleted. The efficacy of VV $\Delta \mathrm{TK} \Delta \mathrm{N} 1 \mathrm{~L}$ as a surgical adjuvant was abrogated only when NK cells were depleted (figure 5B), demonstrating that the proliferation and mobilization of NK, but not $\mathrm{T}$ cells can delay postsurgical mortality in this model. Indeed, analysis of postsurgical adaptive immune responses demonstrates that the antitumor immunity generated on vaccination using $\mathrm{VV} \Delta \mathrm{TK} \Delta \mathrm{N} 1 \mathrm{~L}$ was lost after surgery, further demonstrating that NK cells are the critical component of the efficacy of VV $\Delta \mathrm{TK} \Delta \mathrm{N} 1 \mathrm{~L}$ at preventing postoperative mortality (figure 5C,D). 
To demonstrate broader application of this result, response of orthotopically implanted metastatic 4T1 breast cancer was examined, which again demonstrated a significant prolongation of survival following treatment with $\mathrm{VV} \Delta \mathrm{TK} \Delta \mathrm{N} 1 \mathrm{~L}$ prior to surgical excision (figure $5 \mathrm{E}$ ).

\section{IL-12 can enhance the primary therapeutic and neoadjuvant potential of VV $\Delta T K \Delta N 1 L$}

IL-12 is a pleiotropic cytokine with strong antitumor potential and known effects on both adaptive and innate components on the immune system. We reasoned that by arming VV $\Delta \mathrm{TK} \Delta \mathrm{N} 1 \mathrm{~L}$ with IL-12, the effects of viral therapy on the innate $(\mathrm{NK})$ and adaptive ( $\mathrm{T}$ cell) populations could be augmented, creating a powerful therapeutic for use in primary or neoadjuvant settings. Increased IL-12 expression was detected after viral infection of tumor cell lines with VV $\Delta \mathrm{TK} \Delta \mathrm{N} 1 \mathrm{~L}-\mathrm{mIL} 12$ (expressing murine IL-12) and VV $\Delta \mathrm{TK} \Delta \mathrm{N} 1 \mathrm{~L}-\mathrm{hIL} 12$ (expressing human IL-12) in vitro and the virus retained the capacity to replicate in and kill tumor cells (online supplementary figure S4). In vivo, treatment of DT6606 subcutaneous pancreatic tumors, which responded well to $\mathrm{VV} \Delta \mathrm{TK} \Delta \mathrm{N} 1 \mathrm{~L}$, was dramatically enhanced by the addition of IL-12 to the virus, affecting a $90 \%$ cure rate, compared with $60 \%$ using the unarmed virus (figure 6A,B).

Enhanced efficacy was reflected by improved tumorspecific immunity (figure 6C), increased $\mathrm{T}$ cell infiltration into tumors (figure 6D) and increased activation of splenic CD8+ T cells (figure 6E) demonstrating VV $\Delta \mathrm{T}$ K $\triangle$ N1L-mIl12 as a powerful monotherapeutic agent that can mediate long-term control of tumors by augmentation of adaptive antitumor immune responses.

We next assessed the efficacy of VV $\Delta \mathrm{TK} \Delta \mathrm{N} 1 \mathrm{~L}-\mathrm{mIL} 12$ as a neoadjuvant to surgery using the more aggressive LLC model. Subcutaneous LLC tumors were injected five times daily with VV $\Delta \mathrm{TK} \Delta \mathrm{N} 1 \mathrm{~L}$ or VV $\Delta \mathrm{TK} \Delta \mathrm{N} 1 \mathrm{~L}-\mathrm{mIL} 12$ and the tumor growth monitored. Growth of the primary tumor was controlled significantly better and overall survival improved when the virus was armed with IL-12, demonstrating the value of this addition for presurgical debulking of tumors (figure 7A,B). Encouragingly, when applied as a surgical adjuvant, VV $\Delta$ TK $\Delta$ N1L-mIL12 was able to maintain control of lung metastasis (figure 7C) and significantly extend long-term survival of mice (figure 7D).

We next explored the antitumor efficacy of VV $\Delta \mathrm{T}$ K $\triangle$ N1L-mIL12 in an LY2 murine model of head and neck cancer, representing a model that is intrinsically amenable to neoadjuvant i.t. OV treatment and surgical resection. LY2 models were established subcutaneously in immunocompetent mice and i.t. OV treatment given for 5 days. Six days following the last OV treatment, tumors were excised. Both viruses were able to exert initial control over tumor growth in this model (figure 7E) and OV treatment was able to control both tumor recurrence at the excision site and lymph node metastasis (figure $7 \mathrm{~F}$ ), with VV $\Delta \mathrm{T}$ K $\Delta$ N1L-mIL12 outperforming VV $\Delta \mathrm{TK} \Delta \mathrm{N} 1 \mathrm{~L}$. Importantly, VV $\Delta \mathrm{TK} \Delta \mathrm{N} 1 \mathrm{~L}-\mathrm{mIL} 12$ was able to affect a long-term cure in $80 \%$ of animals (figure $7 \mathrm{G}$ ). We have previously shown that i.t. treatment with an IL-12 expressing OV is non-toxic ${ }^{25}$ and this was confirmed in the LY2 model, in which there was no unresolved perturbation of liver and kidney biochemistry after treatment (online supplementary figure S5). VV $\Delta \mathrm{TK} \Delta \mathrm{N} 1 \mathrm{~L}$ expressing human IL12 (VV $\Delta$ TK $\Delta$ N1L-hIL12), that is fully functional in Syrian hamsters, ${ }^{25}$ was further investigated using a Syrian Hamster subcutaneous model of head and neck cancer. HCPC1 tumors were established and treated as previously prior to surgical excision. These tumors are particularly sensitive to OV treatment and VVATK $\Delta$ N1L was able to affect complete tumor regression prior to surgical excision in 2/10 animals. VV $\Delta$ TK $\Delta$ N1L-hIL12 was able to affect presurgical cure in 5/10 animals. Surgical removal of the remaining tumors affected a complete cure, while 6/9 PBS-treated animals demonstrated tumor recurrence by 15 days post surgery (figure $7 \mathrm{H}$ ).

Overall, these results demonstrate that IL-12 augments the activity of VV $\Delta \mathrm{TK} \Delta \mathrm{N} 1 \mathrm{~L}$ as a monotherapy for primary tumors by enhancing $\mathrm{T}$ cell responses to the tumor. Its potency against aggressive, metastatic tumors is less effective; however, when combined with surgical intervention, it is able to prevent postoperative mortality by preventing recurrence and metastasis that occurs commonly following surgical intervention.

\section{DISCUSSION}

Appropriate activation of the innate immune system is a prerequisite to effective priming of adaptive immune responses against both foreign and self-antigens, including tumor-associated antigen (TAAs). ${ }^{26}$ Viruses are powerful stimulants of the innate immune system and there is an accumulating body of evidence that this effect in its own right may mediate the antitumor effects of some OVs. In a syngeneic colorectal tumor model treated with the Western reserve strain of VV (WRDD), massive tumor necrosis occurred consequent to the influx of neutrophils interrupting tumor vascularity, ${ }^{27}$ while NK cell depletion abrogated tumor clearance following treatment with other OVs. ${ }^{28}$

Animal and clinical studies have demonstrated that surgical manipulation may enhance postoperative metastases. $^{2}{ }^{29}$ Indeed surgical excision of subcutaneously grown LLC tumor has been shown to enhance the rate of lung metastases via removal of an angiogenesis inhibitor secreted by the primary tumor. ${ }^{23}$ Immune suppression is also emerging as a key factor in postsurgical disease spread and components of the innate immune response, including NK responses, are known to be dampened. ${ }^{26} 29$ Surgery-induced immune suppression peaks 3 days post surgery, but the restoration of immune competence may take up to 5 weeks, ${ }^{2}$ generating a significant window of opportunity for tumor dissemination.

At present, there are no FDA-approved perioperative approaches aimed at controlling MRD. Perioperative cytokine therapies using IL-2 (IL-2) ${ }^{30}$ and 
A

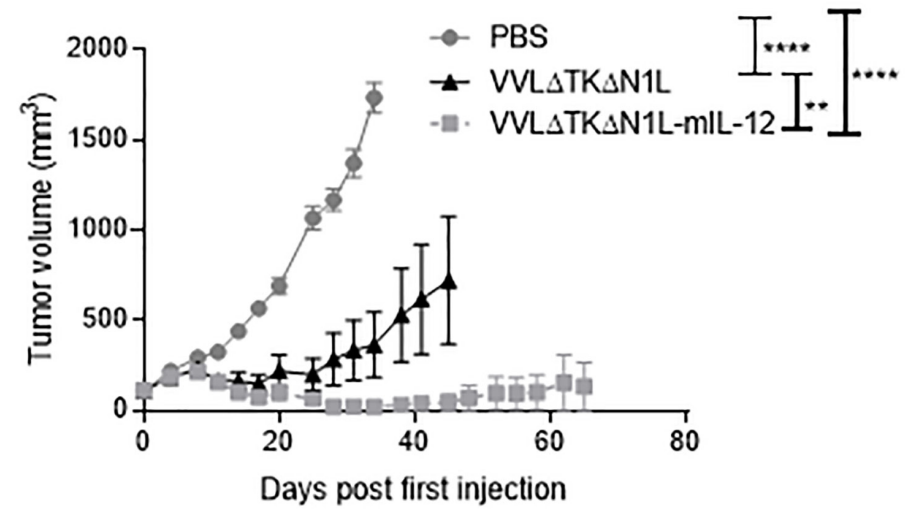

C

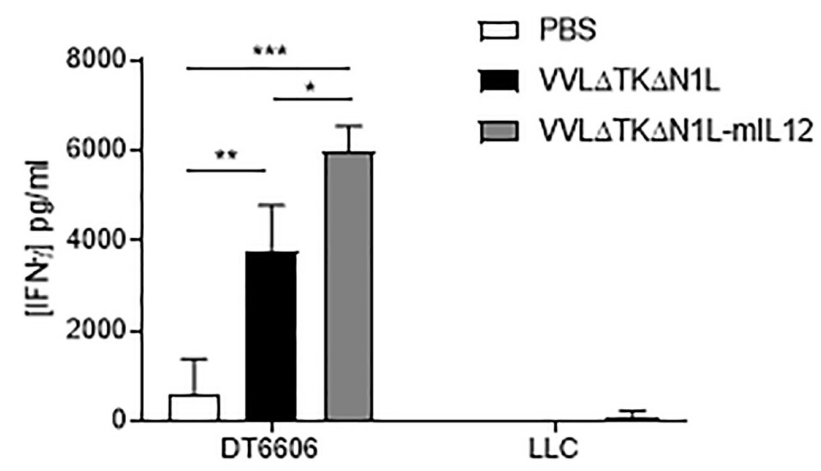

E

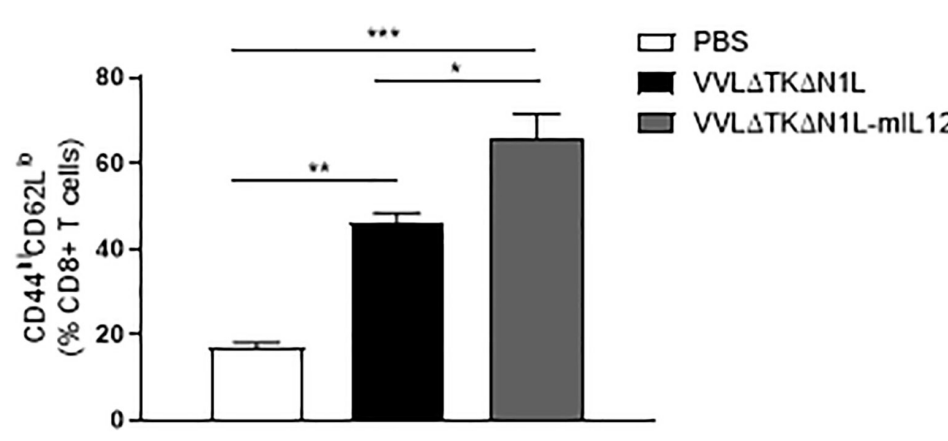

B

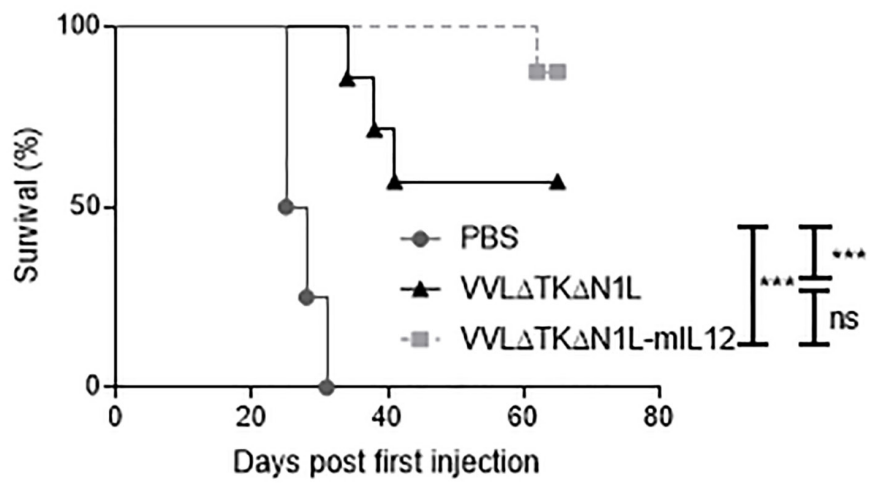

D
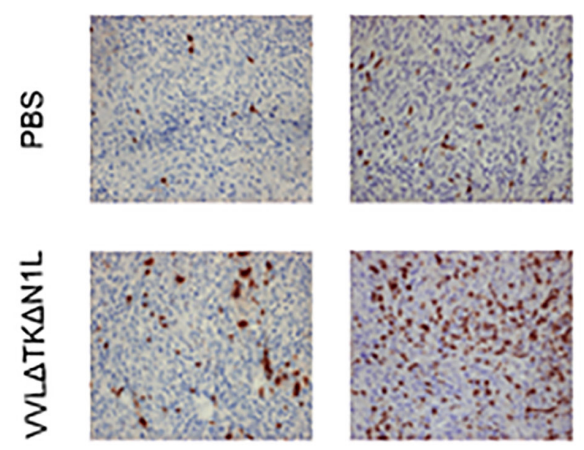

N
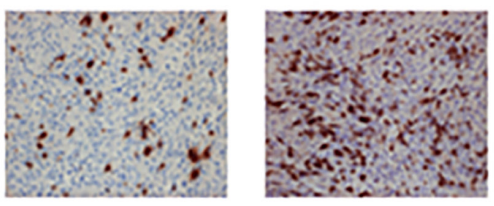

Figure 6 Arming VV $\triangle T K \Delta$ N1L with interleukin (IL)-12 improves efficacy as a monotherapeutic agent. (A)-(E) DT6606 tumors were established in the flanks of immune-competent C57/BI6 mice ( $n=6-7 /$ group). Once palpable, mice were injected intratumoral with $1 \times 10^{8} \mathrm{PFU}$ VV $\triangle T K \triangle N 1 \mathrm{~L}, \mathrm{VV} \triangle \mathrm{TK} \triangle \mathrm{N} 1 \mathrm{~L}-\mathrm{mL} 12$ or PBS daily for 5 days. (A) Tumor growth was monitored and significance at each time point analyzed using two-way analysis of variance (ANOVA) with post hoc Tukey tests. Significance at day 34 is shown. (B) Kaplan-Meier survival analysis with log rank (Mantel-Cox) tests was used to assess survival. (C) DT6606 flank tumors were treated as above ( $n=3-4$ /group) and 14 days after the first treatment splenocytes were analyzed for response to growth-arrested Lewis lung carcinoma (LLC) or DT6606 (control) tumor cells ex vivo using interferon $\gamma$ (IFN $\gamma$ ) ELISA after 72 hours coculture. (D) DT6606 tumors were treated as above and 14 days after the first treatment, tumor sections were immunostained with CD4 or CD8 antibodies ( $n=3-4$ /group). Representative images are shown (magnification $\times 200$ ) and a manual cell count pre-HPF (taken from 15 HPFs) depicted graphically. One-way ANOVA with post hoc Tukey tests was used to assess significance. (E) DT6606 tumors were treated as above ( $n=3-4 /$ group) and 14 days after the first treatment, splenocytes analyzed using FACS for activated CD8+ T cells using CD44 and CD62L (of live, CD45+, CD3+, CD8+ populations). One-way ANOVA with post hoc Tukey tests were used to assess significance. In all cases, the mean \pm SEM is shown. ${ }^{*} p<0.05 ;{ }^{* *} p<0.01$; ${ }^{* * *} \mathrm{p}<0.001$. 


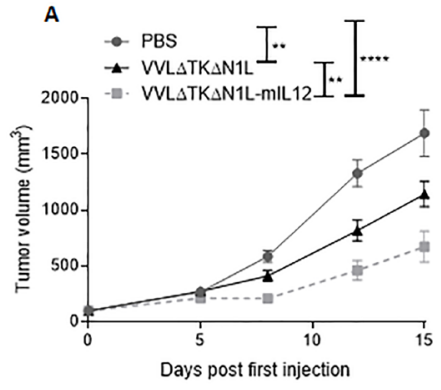

B

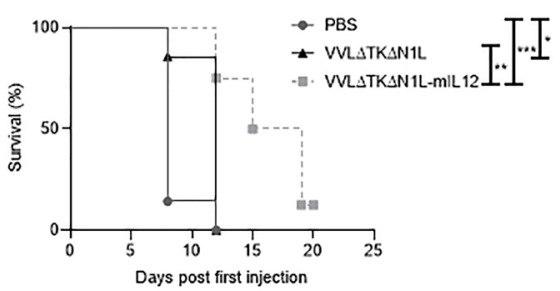

c
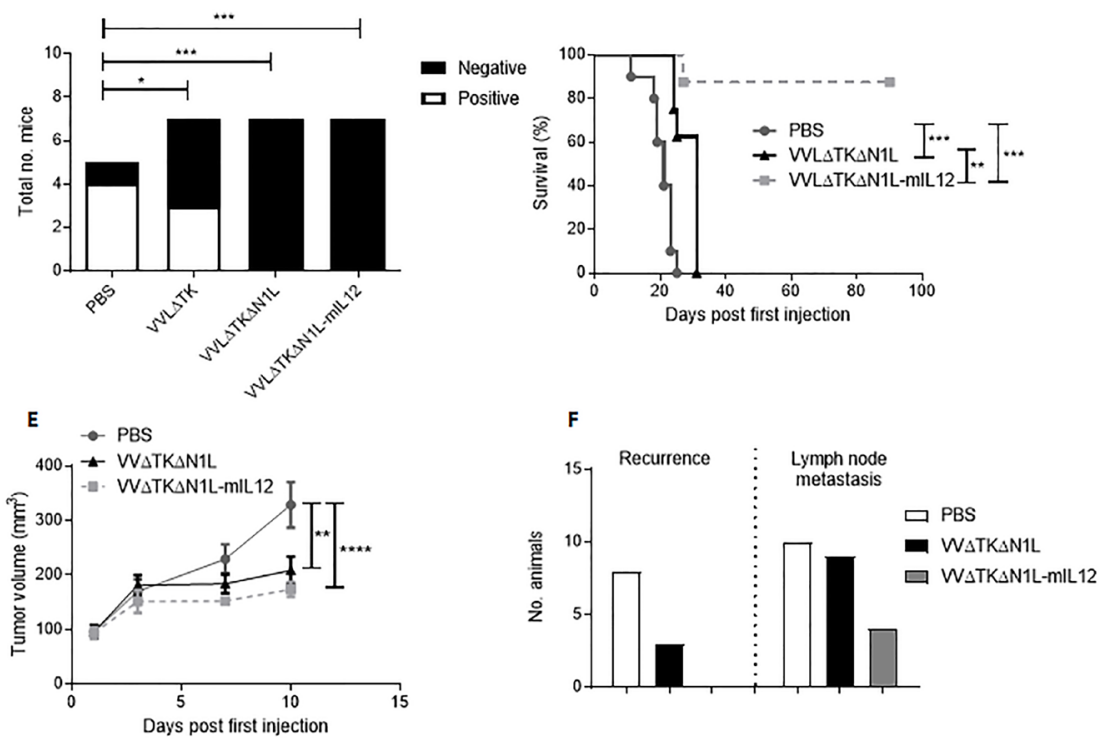

G

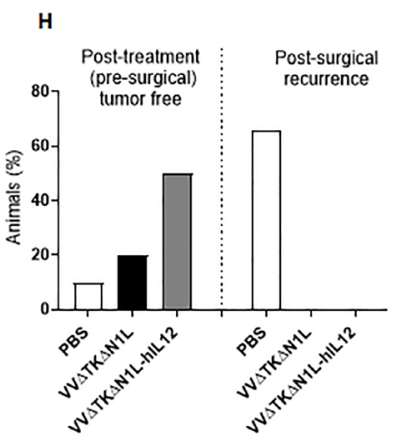

Figure $7 \quad$ VV $\Delta T K \Delta$ N1L-IL12 is an effective surgical adjuvant treatment in vivo. (A) Lewis lung carcinoma (LLC) tumors were established in the flanks of immune-competent C57/BI6 mice ( $\mathrm{n}=7 /$ group). Once palpable, mice were injected intratumoral (i.t) with $1 \times 10^{8} \mathrm{PFU} V \mathrm{~V} \triangle \mathrm{TK} \Delta \mathrm{N} 1 \mathrm{~L}, \mathrm{VV} \triangle \mathrm{TK} \Delta \mathrm{N} 1 \mathrm{~L}-\mathrm{mIL} 12$ or PBS daily for 5 days ( $\left.\mathrm{n}=8-10 / \mathrm{group}\right)$. (A) Tumor growth was monitored until resection. A two-way analysis of variance (ANOVA) with post hoc Tukey tests was used to assess significance at each time point. (B) Overall survival was monitored using Kaplan-Meier survival analysis with log rank (Mantel-Cox) tests. (C) LLC tumors were treated as above. Tumors were surgically resected 7 days after the final dose of virus. Mice were sacrificed at day 15 , H\&E stained and analyzed for the presence of metastasis by a pathologist blinded to treatment groups. Lungs were scored as negative or positive and a Fishers exact test used to determine significance. (D) Mice treated in (C) were assessed for long-term survival after treatment and surgical excision of tumors using Kaplan-Meier survival analysis with log rank (Mantel-Cox) tests. (E) LY2 murine head and neck tumors were established in the flanks of immune-competent C57/Bl6 mice ( $\mathrm{n}=10 / \mathrm{group})$. Once palpable, mice were injected i.t with $1 \times 10^{8} \mathrm{PFU}$ VV $\triangle T K \Delta N 1 \mathrm{~L}$, VV $\triangle T K \Delta N 1 \mathrm{~L}-\mathrm{mIL} 12$ or PBS daily for 5 days. Tumor growth was monitored until resection 6 days following the last treatment. A two-way ANOVA with post hoc Tukey tests was used to assess significance at each time point. Significance at day 10 is shown. (F) LY2 tumors were treated as above and recurrence at the excision site or lymph node metastasis on sacrifice recorded. (G) LY2 tumors were treated as above and survival post resection monitored using Kaplan-Meier survival analysis with log rank (Mantel-Cox) tests. (H) Hamster HCPC1 tumors were established in the flank of Syrian hamsters. Once palpable, hamsters were injected i.t with $1 \times 10^{8} \mathrm{PFU}$ VV $\Delta T K \Delta N 1 \mathrm{~L}, \mathrm{VV} \Delta T K \Delta N 1 \mathrm{~L}-\mathrm{hIL} 12$ or PBS daily for 5 days ( $n=10 /$ group). The effect of treatment on the primary tumor is shown. Six days following the last treatment, remaining tumors were surgically excised (PBS $n=9$; VV $\Delta T K \Delta N 1 L n=8$; VV $\Delta T K \Delta N 1 L-h I L 12 n=5$ ) and recurrence monitored. In all cases, the mean \pm SEM is shown. ${ }^{*} p<0.05 ;{ }^{* *} p<0.01 ;{ }^{* \star *} p<0.001 ;{ }^{* \star \star} p<0.0001$. 
interferon- $\alpha$ (IFN- $\alpha)^{31}$ have both shown encouraging effects by preventing postoperative NK suppression; however, these therapies have not been developed further due to substantial dose-limiting toxicities. ${ }^{32}$ Therefore, development of neoadjuvant therapeutic regimes for prevention of recurrence and metastasis after surgery remains an important area of unmet clinical need.

Neoadjuvant virotherapy has the potential to aid eradication of in situ microscopic disease. Tai et al have demonstrated that a single intravenous dose of replicating pox virus administered prior to surgery could reverse surgical stress-induced NK cell suppression as well as the associated enhanced metastases in experimental models of metastatic breast cancer and melanoma. ${ }^{6}$ A similar result was also obtained with inactivated influenza vaccine ${ }^{33}$ and maraba virus, ${ }^{34}$ all mediated through enhanced NK cellular activity. More recently, the role of Maraba and other OV, although notably not VV, in control of postsurgical breast cancer recurrence has also been reported. ${ }^{35} 36$ These studies are encouraging, but as yet lack long-term survival data. Here, we demonstrate rational modification of the $\mathrm{VV}$ genome that promotes its efficacy as both a primary therapeutic agent via improved activation of adaptive immunity and as a neoadjuvant agent via improved activation of innate immunity required for postsurgical control of disease.

Most viruses have acquired strategies to ensure survival, replication and propagation in their hosts. These are mediated by different virulence gene products and in VV the N1L gene product has been shown to be vital for inhibition of innate immune responses against the virus. ${ }^{37}$ The N1L product has been demonstrated as a potent inhibitor of NF- $\mathrm{KB}$ signalling and thus inflammation required for T cell activation. ${ }^{16}$ We reasoned that deletion of the N1L gene from VV may serve to locally enhance the innate antiviral immune responses consequent to viral oncolysis and that i.t., as opposed to systemic, injection of the tumor bed prior to excision would allow for repeat dosing that provides a powerful mechanism by which to activate local immune responses required for prevention of postsurgical tumor outgrowth.

Using a panel of murine and human tumor cell lines, we found that VVL15 $\Delta \mathrm{N} 1 \mathrm{~L}$ was at least as potent, if not more so than N1L-intact VV (VV $\Delta \mathrm{TK}$ ) at killing murinederived cancer cells in vitro, a finding that may not be surprising given the potential antiapoptotic function of the N1L protein. ${ }^{38}$

Here, we demonstrate that i.t.-administered VV $\Delta \mathrm{T}$ $\mathrm{K} \Delta \mathrm{N} 1 \mathrm{~L}$ was able to reduce metastases of LLC tumors from the primary site. Possible explanations for this effect could include blockage of peritumor vasculature by enhanced leukocyte infiltration, enhanced numbers and activity of circulating NK cells and/or enhanced antitumor immune surveillance. i.t. neutrophil infiltration following virotherapy has previously been reported ${ }^{27}$ and VV $\Delta \mathrm{TK} \Delta \mathrm{N} 1 \mathrm{~L}$ appeared to enhance this response. The prolonged presence of neutrophils within the TME has been associated with tumor progression via multiple mechanisms $^{39}$ and it is interesting to note that $\mathrm{KC}$, a murine neutrophil chemoattractant, was constitutively secreted by LLC tumor cells in vitro. Analogous to GMCSF, in the resting state, $\mathrm{KC}$ may act as an autocrine growth factor for some tumor cells. By contrast, recruitment and stimulation of neutrophils, as occurs following virotherapy, have also been associated with tumor cell cytotoxicity and suppression. ${ }^{40}$ The latter is attributed to mechanisms such the mechanical blockage of tumor vessels, excess release of cytotoxic agents, enhanced antigen-dependent cellular cytotoxicity in the presence of antitumor antibodies, or indeed through a phenotypic switch of neutrophils to being able to present scavenged tumor antigens ${ }^{41}$ and an in-depth analysis of the neutrophil phenotype induced by VV $\Delta T K \Delta N 1 \mathrm{~L}$ is warranted to determine the extent to which neutrophil infiltration controls tumor progression.

A screen of inflammatory cytokines and chemokines within virally infected LLC flank tumors revealed the selective enhancement of KC, GCSF, IL $1 \alpha / \beta$ and MIP1 $\alpha$ expression. These are all regulated by NF- $\kappa B$ transcription factors and their expression might have been expected to be enhanced given the suppressive effect of the N1L protein on this pathway. ${ }^{37}$ Based on their wellcharacterized functions, enhanced production of $\mathrm{KC}$ (mainly from infected tumor cells) and GCSF was most likely to be responsible for the enhanced infiltration of i.t. neutrophils seen in vivo following VV $\Delta \mathrm{TK} \Delta \mathrm{N} 1 \mathrm{~L}$ infection. These neutrophils, in turn, are likely a major source of IL1 $\beta$ and MIP1 $\alpha$ in vivo, which display potent chemotactic activity for macrophages, dendritic, NK and T cells. ${ }^{42}$

VVATK $\triangle$ N1L infection upregulates the expression of the IL1 family of cytokines from both DCs and macrophages: IL1 $\alpha$, IL1 $\beta$ and IL18. The latter two are surrogate markers for the activation of inflammasome platforms, a group of multimeric protein complexes that form in response to a range of exogenous and endogenously generated pathogen-associated or damage-associated molecules. ${ }^{43}$ Activated caspase-1 within the inflammasome is responsible for cleavage and activation of IL1 $\beta$ and IL18 precursor molecules. Although transcription of the precursor pro-IL1 $\beta$ gene is under the control of NF-KB transcription factors, pro-IL18 mRNA is constitutively expressed and has different regulatory controls. ${ }^{43}$ The fact that in our studies IL18 was also upregulated by VVL15 $\Delta$ N1L infection suggests that the N1L protein may additionally regulate inflammasome signaling by a previously undescribed mechanism of action. This warrants further investigation.

In comparison to VV $\Delta \mathrm{TK}, \mathrm{VV} \Delta \mathrm{TK} \Delta \mathrm{N} 1 \mathrm{~L}$ infection enhanced the global activation of DCs and macrophages and in the latter also enhanced the upregulation of MHCII, effectively transforming them into professional APCs. It has been reported that human IL-8, the murine $\mathrm{KC}$ analogue, has the capacity to retain DCs in the tumor, preventing trafficking to lymphoid organs for antigen presentation. ${ }^{44}$ Here, we found that in vivo, i.t.-delivered 
VVL15 $\Delta$ N1L was able to enhance the percentage of DCs in murine spleens, but further analysis of their phenotype would be important for translation of this therapy. Given the effects of VV $\Delta \mathrm{TK} \Delta \mathrm{N} 1 \mathrm{~L}$ on innate immune populations, particularly those considered important for controlling postsurgical metastasis, we postulated that VV $\Delta \mathrm{TK} \Delta \mathrm{N} 1 \mathrm{~L}$ might be an effective neoadjuvant to conventional surgery for tumor treatment. In order to assess this, we analyzed different surgical models in the neoadjuvant setting. Tumors were excised 5-7 days after the final dose of virus to allow mice to recover from the effects of a protracted course of viral therapy and to provide adequate time for the development of an antitumor adaptive immune response. Importantly, our experiments have demonstrated that an elevated systemic NK response is sustained for at least 2 weeks following i.t. virus and therefore should last throughout the critical perioperative period. The efficacy results demonstrated a significant survival benefit for mice pretreated with VV $\Delta \mathrm{TK} \Delta \mathrm{N} 1 \mathrm{~L}$ prior to surgical excision of their primary tumor. This effect was mediated by the enhanced elevation of NK cells, evidenced by the complete abrogation of response when the experiment was repeated in mice in which NK cells were depleted.

Preclinical and clinical data suggest that the efficacy of OV is significantly improved by arming the virus with an immunomodulatory gene, to encourage destruction of the immune-suppressive environment of the tumor and promote long-term antitumor surveillance. IL-12 has emerged as one of the most promising cytokines for antitumor therapeutics. Importantly, in the context of neoadjuvant therapy, it is a potent activator of both NK and T cell IFN- $\gamma$ production, ${ }^{45}$ has been demonstrated to have antiangiogenic properties ${ }^{46}$ and can also upregulate cellular expression of MHCI and MHCII molecules, ${ }^{47}$ facilitating presentation of tumor antigens important for long-term disease surveillance. However, its use has been limited by the severe toxicity induced on systemic administration. Use in a neoadjuvant setting however, by i.t. administration prior to surgery, circumvents this toxicity and we have demonstrated that i.t. administration of IL-12 in the context of OV is a safe and effective way to enhance the effects of VV $\Delta \mathrm{TK} \Delta \mathrm{N} 1 \mathrm{~L}$ as both a primary therapeutic agent and as a neoadjuvant agent. Indeed, neoadjuvant treatment with VV $\Delta T K \Delta$ N1L-m/ hIL12 completely prevented postoperative recurrence in vivo using head and neck cancer models that are intrinsically amenable to i.t. injection of virus, suggesting presurgical treatment with IL-12 armed VV can induce robust antitumor immune responses that significantly extend mortality. Interestingly, the power of immune checkpoint inhibitor (ICI) molecules as neoadjuvant agents with the puropse of inducing antitumor $\mathrm{T}$ cell reponses prior to surgical excision has been investigated clinically, with some pathological responses shown. ${ }^{48} \mathrm{OV}$ such as TVEC and VV have been demonstrated to induce CD8 +T cell infiltration into tumors and upregulate expression of CTLA4, PD-L1 and other immune checkpoint molecules which would normally block T cell activation ${ }^{35} 3649$ and as such can sensitize tumors to ICI, ${ }^{50}$ suggesting that neoadjuvant treatment with VV $\Delta$ TK $\Delta$ N1L-IL12 would have an additional advantage of ICI synergy, which warrants further preclinical evaluation.

Although these studies were carried out using surgical excision of flank tumors, representing a tumor and immune environment less complex than would occur in the clinical scenario, the principles demonstrated suggest that VV $\Delta \mathrm{TK} \Delta \mathrm{N} 1 \mathrm{~L}-\mathrm{IL} 12$ is a rationally engineered viral platform with particular efficacy as a neoadjuvant to oncological surgery, where its properties may minimize postoperative solid tumor metastasis and recurrence, significantly prolonging survival.

Acknowledgements The authors are grateful to David Tuveson and Istvan Fodor for providing materials for this study.

Contributors YW conceived and supervized this study. JA and LSC designed the detailed experiments. JA conducted most of the experiments and analyzed results with LSC. MY helped viral construction. JW, AH, YL, HL, ZZ, SL and DG performed the in vivo animal experiments. CAY and JS characterized the murine head and neck cancer LY2 in vivo model. NL interpreted some experiments and critically reviewed the manuscript. YC prepared the viruses. LSC, JA and YW interpreted all results and wrote the manuscript.

Funding This project was supported by the Wellcome Trust Research Training Fellowship (097983/Z/11/Z), Nature Sciences Foundation of China (U170420035 and 81771776), National Key R\&D Program of China (2016YFE0200800) and the core funding for development of the Cell and Gene Therapy Programme by Zhengzhou University. LSC is funded by The MRC ((MR/M015696/1).

Competing interests None declared.

Patient consent for publication Not required.

Ethics approval All mouse studies were carried out under the terms of the Home Office Project Licence PPL 70/6030 and subject to Queen Mary University of London ethical review, according to the guidelines for the welfare and use of animals in cancer research. Mouse and Syrian hamster procedures performed in this study were approved by the Animal Welfare and Research Ethics Committee of Zhengzhou University (Zhengzhou, China).

Provenance and peer review Not commissioned; externally peer reviewed.

Data availability statement Data sharing not applicable as no datasets generated and/or analyzed for this study. All data are published in this paper. Materials are available upon reasonable reqest based on an appropriate MTA.

Open access This is an open access article distributed in accordance with the Creative Commons Attribution 4.0 Unported (CC BY 4.0) license, which permits others to copy, redistribute, remix, transform and build upon this work for any purpose, provided the original work is properly cited, a link to the licence is given, and indication of whether changes were made. See https://creativecommons.org/ licenses/by/4.0/.

ORCID iD

Yaohe Wang http://orcid.org/0000-0003-2367-6313

\section{REFERENCES}

1 Johnson DB, Puzanov I, Kelley MC. Talimogene laherparepvec (T-VEC) for the treatment of advanced melanoma. Immunotherapy 2015;7:611-9.

2 Coffey JC, Wang JH, Smith MJF, et al. Excisional surgery for cancer cure: therapy at a cost. Lancet Oncol 2003;4:760-8.

3 Klein CA, Blankenstein TJF, Schmidt-Kittler O, et al. Genetic heterogeneity of single disseminated tumour cells in minimal residual cancer. Lancet 2002;360:683-9.

4 Baum M, Chaplain MA, Anderson AR, et al. Does breast cancer exist in a state of chaos? Eur J Cancer 1999;35:886-91.

5 Tai L-H, Tanese de Souza C, Sahi S, et al. A mouse tumor model of surgical stress to explore the mechanisms of postoperative 
immunosuppression and evaluate novel perioperative immunotherapies. J Vis Exp 2014;85. doi:10.3791/51253

6 Tai L-H, de Souza CT, Bélanger S, et al. Preventing postoperative metastatic disease by inhibiting surgery-induced dysfunction in natural killer cells. Cancer Res 2013;73:97-107.

7 Filley AC, Dey M. Immune system, friend or foe of oncolytic virotherapy? Front Oncol 2017:7:106.

8 Chard LS, Maniati E, Wang $P$, et al. A vaccinia virus armed with interleukin-10 is a promising therapeutic agent for treatment of murine pancreatic cancer. Clin Cancer Res 2015;21:405-16.

9 Bartal I, Melamed R, Greenfeld K, et al. Immune perturbations in patients along the perioperative period: alterations in cell surface markers and leukocyte subtypes before and after surgery. Brain Behav Immun 2010;24:376-86.

10 Hiley CT, Chard LS, Gangeswaran R, et al. Vascular endothelial growth factor A promotes vaccinia virus entry into host cells via activation of the Akt pathway. J Virol 2013;87:2781-90.

11 Lee J-W, Shahzad MMK, Lin YG, et al. Surgical stress promotes tumor growth in ovarian carcinoma. Clin Cancer Res 2009;15:2695-702.

12 Hughes J, Wang P, Alusi G, et al. Lister strain vaccinia virus with thymidine kinase gene deletion is a tractable platform for development of a new generation of oncolytic virus. Gene Ther 2015;22:476-84.

13 Gratz MS, Suezer Y, Kremer M, et al. N1L is an ectromelia virus virulence factor and essential for in vivo spread upon respiratory infection. J Virol 2011;85:3557-69.

14 Jacobs N, Bartlett NW, Clark RH, et al. Vaccinia virus lacking the $\mathrm{Bcl}-2$-like protein $\mathrm{N} 1$ induces a stronger natural killer cell response to infection. J Gen Virol 2008;89:2877-81.

15 Bartlett N, Symons JA, Tscharke DC, et al. The vaccinia virus N1L protein is an intracellular homodimer that promotes virulence. J Gen Virol 2002;83:1965-76.

16 Ren $\mathrm{H}$, Ferguson BJ, Maluquer de Motes $\mathrm{C}$, et al. Enhancement of CD8(+) T-cell memory by removal of a vaccinia virus nuclear factorкB inhibitor. Immunology 2015;145:34-49.

17 Lasek W, Zagożdżon R, Jakobisiak M. Interleukin 12: still a promising candidate for tumor immunotherapy? Cancer Immunol Immunother 2014:63:419-35.

18 Dénes B, Yu J, Fodor N, et al. Suppression of hyperglycemia in NOD mice after inoculation with recombinant vaccinia viruses. Mol Biotechnol 2006;34:317-28.

19 Wang Z, Martinez J, Zhou W, et al. Modified H5 promoter improves stability of insert genes while maintaining immunogenicity during extended passage of genetically engineered MVA vaccines. Vaccine 2010;28:1547-57.

20 Yuan M, Gao X, Chard LS, et al. A marker-free system for highly efficient construction of vaccinia virus vectors using CRISPR Cas9. Mol Ther Methods Clin Dev 2015;2:15035.

21 Griffith JW, Sokol CL, Luster AD. Chemokines and chemokine receptors: positioning cells for host defense and immunity. Annu Rev Immunol 2014;32:659-702.

22 Zitvogel L, Kepp O, Galluzzi L, et al. Inflammasomes in carcinogenesis and anticancer immune responses. Nat Immunol 2012;13:343-51.

23 O'Reilly MS, Holmgren L, Shing Y, et al. Angiostatin: a novel angiogenesis inhibitor that mediates the suppression of metastases by a Lewis lung carcinoma. Cell 1994;79:315-28.

24 Wang H-L, Ning T, Li M, et al. Effect of endostatin on preventing postoperative progression of distant metastasis in a murine lung cancer model. Tumori 2011:97:787-93.

25 Wang P, Li X, Wang J, et al. Re-designing interleukin-12 to enhance its safety and potential as an anti-tumor immunotherapeutic agent. Nat Commun 2017:8:1395.

26 Schenten D, Medzhitov R. The control of adaptive immune responses by the innate immune system. Adv Immunol 2011;109:87-124.

27 Breitbach CJ, Paterson JM, Lemay CG, et al. Targeted inflammation during oncolytic virus therapy severely compromises tumor blood flow. Mol Ther 2007;15:1686-93.
28 Prestwich RJ, Errington F, Diaz RM, et al. The case of oncolytic viruses versus the immune system: waiting on the judgment of Solomon. Hum Gene Ther 2009;20:1119-32.

29 Lejeune FJ. Is surgical trauma prometastatic? Anticancer Res 2012;32:947-51.

30 Deehan DJ, Heys SD, Ashby J, et al. Interleukin-2 (IL-2) augments host cellular immune reactivity in the perioperative period in patients with malignant disease. Eur J Surg Oncol 1995;21:16-22.

31 Houvenaeghel G, Bladou F, Blache JL, et al. Tolerance and feasibility of perioperative treatment with interferon-alpha $2 \mathrm{~A}$ in advanced cancers. Int Surg 1997;82:165-9.

32 Dranoff G. Cytokines in cancer pathogenesis and cancer therapy. Nat Rev Cancer 2004:4:11-22.

33 Tai L-H, Zhang J, Auer RC. Preventing surgery-induced NK cell dysfunction and cancer metastases with influenza vaccination. Oncoimmunology 2013;2:e26618.

34 Zhang J, Tai L-H, Ilkow CS, et al. Maraba MG1 virus enhances natural killer cell function via conventional dendritic cells to reduce postoperative metastatic disease. Mol Ther 2014;22:1320-32.

35 Bommareddy PK, Shettigar M, Kaufman HL. Integrating oncolytic viruses in combination cancer immunotherapy. Nat Rev Immunol 2018;18:498-513.

36 Kleinpeter $\mathrm{P}$, Fend $\mathrm{L}$, Thioudellet $\mathrm{C}$, et al. Vectorization in an oncolytic vaccinia virus of an antibody, a Fab and a scFv against programmed cell death -1 (PD-1) allows their intratumoral delivery and an improved tumor-growth inhibition. Oncoimmunology 2016;5:e1220467.

37 Smith GL, Benfield CTO, Maluquer de Motes C, et al. Vaccinia virus immune evasion: mechanisms, virulence and immunogenicity. J Gen Virol 2013;94:2367-92.

38 Cooray S, Bahar MW, Abrescia NGA, et al. Functional and structural studies of the vaccinia virus virulence factor $\mathrm{N} 1$ reveal a $\mathrm{Bcl}-2$-like anti-apoptotic protein. J Gen Virol 2007;88:1656-66.

39 Gregory AD, Houghton AM. Tumor-Associated neutrophils: new targets for cancer therapy. Cancer Res 2011;71:2411-6.

40 Colombo MP, Lombardi L, Stoppacciaro A, et al. Granulocyte colony-stimulating factor (G-CSF) gene transduction in murine adenocarcinoma drives neutrophil-mediated tumor inhibition in vivo. neutrophils discriminate between G-CSF-producing and G-CSFnonproducing tumor cells. J Immunol 1992;149:113-9.

41 Matsushima H, Geng S, Lu R, et al. Neutrophil differentiation into a unique hybrid population exhibiting dual phenotype and functionality of neutrophils and dendritic cells. Blood 2013;121:1677-89.

42 Scapini P, Lapinet-Vera JA, Gasperini S, et al. The neutrophil as a cellular source of chemokines. Immunol Rev 2000;177:195-203.

43 Latz E, Xiao TS, Stutz A. Activation and regulation of the inflammasomes. Nat Rev Immunol 2013;13:397-411.

44 Alfaro C, Suárez N, Martínez-Forero I, et al. Carcinoma-Derived interleukin-8 disorients dendritic cell migration without impairing Tcell stimulation. PLoS One 2011;6:e17922.

45 Manetti R, Parronchi P, Giudizi MG, et al. Natural killer cell stimulatory factor (interleukin 12 [IL-12]) induces T helper type 1 (Th1)-specific immune responses and inhibits the development of IL-4-producing Th cells. J Exp Med 1993;177:1199-204.

46 Coughlin CM, Salhany KE, Gee MS, et al. Tumor cell responses to IFNgamma affect tumorigenicity and response to IL-12 therapy and antiangiogenesis. Immunity 1998;9:25-34.

47 Weiss JM, Subleski JJ, Wigginton JM, et al. Immunotherapy of cancer by IL-12-based cytokine combinations. Expert Opin Biol Ther 2007;7:1705-21

48 Blank CU, Rozeman EA, Fanchi LF, et al. Neoadjuvant versus adjuvant ipilimumab plus nivolumab in macroscopic stage III melanoma. Nat Med 2018;24:1655-61.

49 Ribas A, Dummer R, Puzanov I, et al. Oncolytic virotherapy promotes intratumoral $T$ cell infiltration and improves anti-PD-1 immunotherapy. Cell 2017;170:1109-19.

50 Chon $\mathrm{HJ}$, Lee WS, Yang $\mathrm{H}$, et al. Tumor microenvironment remodeling by intratumoral oncolytic vaccinia virus enhances the efficacy of Immune-Checkpoint blockade. Clin Cancer Res 2019;25:1612-23. 


\section{Correction: A new oncolytic Vaccinia virus augments antitumor immune responses to prevent tumor recurrence and metastasis after surgery}

Ahmed J, Chard LS, Yuan M, et al. A new oncolytic Vaccinia virus augments antitumor immune responses to prevent tumor recurrence and metastasis after surgery. J ImmunoTher Cancer 2020;8:e00415. doi: 10.1136/jitc-2019-000415

In this article, the author Joel Schwartz was incorrectly linked to affiliation 3 twice and authors Nicholas Lemoine and Yaohe Wang should be linked to affiliation 2 in addition to affiliation 1 . The correct author and affiliation list are shown below:

Jahangir Ahmed, ${ }^{1}$ Louisa S Chard, ${ }^{1}$ Ming Yuan, ${ }^{1}$ Jiwei Wang, ${ }^{2}$ Anwen Howells, ${ }^{1}$ Yuenan $\mathrm{Li}^{2}{ }^{2}$ Haoze Li, ${ }^{2}$ Zhongxian Zhang, ${ }^{2}$ Shuangshuang Lu, ${ }^{2}$ Dongling Gao, ${ }^{2}$ Pengju Wang, ${ }^{2}$ Yongchao Chu, ${ }^{2}$ Chadwan Al Yaghchi, ${ }^{1}$ Joel Schwartz, ${ }^{3}$ Ghassan Alusi, ${ }^{1}$ Nicholas Lemoine, ${ }^{1,2}$ Yaohe Wang ${ }^{1,2}$

${ }^{1}$ Centre for Biomarkers \& Biotherapeutics, Barts Cancer Institute, Queen Mary University of London, London, UK

${ }^{2}$ National Centre for International Research in Cell and Gene Therapy, Zhengzhou University, Zhengzhou, Henan, China

${ }^{3}$ University of Illinois at Chicago, Chicago, Illinois, USA

Open access This is an open access article distributed in accordance with the Creative Commons Attribution 4.0 Unported (CC BY 4.0) license, which permits others to copy, redistribute, remix, transform and build upon this work for any purpose, provided the original work is properly cited, a link to the licence is given, and indication of whether changes were made. See https://creativecommons.org/licenses/by/4.0/.

@ C Author(s) (or their employer(s)) 2020. Re-use permitted under CC BY. Published by BMJ.

J Immunother Cancer 2020;8:e000415corr1. doi:10.1136/jitc-2019-000415corr1

A) Check for updates 